\title{
Definition of center of mass for isolated physical systems and unique foliations by stable spheres with constant mean curvature
}

\author{
Gerhard Huisken ${ }^{1}$, Shing-Tung Yau ${ }^{2}$ \\ ${ }^{1}$ Mathematische Fakultät, Universität Tübingen, Auf der Morgenstelle 10, D-72076 Tübingen, \\ Germany \\ 2 Department of Mathematics, Harvard University, One Oxford Street, Cambridge, MA 02138, \\ USA \\ Oblatum 6-VIII-1995 \& 14-VIII-1995
}

to Reinhold Remmert

In the description of isolated gravitating systems in General Relativity a spacelike timeslice has the structure of a complete Riemannian three-manifold with an asymptotically flat end. Let $(N, g)$ denote an end of such a complete Riemannian three-manifold, i.e. $N$ is diffeomorphic to $\mathbf{R}^{3} \backslash B_{1}(0)$, and the metric on $N$ asymptotically approaches the Euclidean metric near infinity:

$$
g_{i j}=\left(1+\frac{2 m}{r}\right) \delta_{i j}+O\left(r^{-2}\right)
$$

where $r$ denotes Euclidean distance in $\mathbf{R}^{3}$. The constant $m$ can be interpreted as the total mass of the isolated system in the end $N$ and is refered to as ADM - mass in the physical literature, compare [ADM].

A basic version of the positive mass theorem [SY1,SY2] states that $m$ is nonnegative if $N$ is the end of a complete three-manifold with nonnegative scalar curvature. Moreover, the mass $m$ is strictly positive unless the threemanifold is flat. It has also been established in [B1] that the mass can be geometrically defined independent of a particular coordinate system.

In this paper we define a geometric center of mass at infinity for asymptotically flat ends with strictly positive mass $m>0$ by constructing a unique constant mean curvature foliation in the exterior region.

After some auxiliary results in Sect. 1 we introduce and investigate a class of "round" surfaces in Sect. 2, in which the constant mean curvature surfaces are to be constructed. The existence proof in Sect. 3 uses a heatflow method to deform a coordinate sphere in normal direction, decreasing its isoperimetric ratio until the surfaces approach a constant mean curvature surface. More precisely, given a coordinate sphere $F_{0}: S^{2} \rightarrow N$ we solve the initial value problem 


$$
\begin{aligned}
\frac{d}{d t} F(p, t) & =(h-H)(p, t) v(p, t) \quad p \in S^{2}, \quad t>0 \\
F(p, 0) & =F_{0}(p),
\end{aligned}
$$

where $H$ and $v$ are the mean curvature and the unit normal on the moving hypersurface respectively and $h=\oint H d \mu$ is the average of the mean curvature. This evolution decreases area while fixing the enclosed volume, and we prove that for sufficiently large initial spheres the solution exists for all $t>0$ and converges to a constant mean curvature sphere as $t \rightarrow \infty$. In this part of the proof the assumption $m>0$ enters crucially when showing that the surfaces do not drift off to infinity during the evolution.

In Sect. 4 we use the positivity of the mass again to prove that the so constructed surfaces form a stable constant mean curvature foliation for an exterior region of $N$, approaching a family of concentric Euclidean spheres. The foliation can be interpreted as a geometric "center of mass" for the infinitely far observer. It defines a natural coordinate system near infinity in an intrinsic way, while retaining regularity and decay properties near infinity that were extensively used in previous studies of foliations near infinity, see e.g. [B2] and $[\mathrm{CK}]$.

In Sect. 5 we prove uniqueness of the foliation for ends $N$ with positive mass. More precisely, we prove that the leaves of the foliation are the only stable constant mean curvature surfaces outside a small interior region, compare Theorem 5.1.

\section{Preliminaries}

Let $N$ be a three-dimensional Riemannian manifold diffeomorphic to $\mathbf{R}^{3} \backslash B_{1}(0)$ with a Euclidean coordinate system $\left\{y_{\alpha}\right\}$. Greek indices run from 1 to 3 and we write $r=\left(\sum_{1}^{3} y_{\alpha}^{2}\right)^{\frac{1}{2}}$ for the Euclidean distance. We assume that the metric $\bar{g}=\left\{\bar{g}_{\alpha \beta}\right\}$ is asymptotically flat, i.e. for $r \geqq 1$ we have

$$
\bar{g}_{\alpha \beta}=\left(1+\frac{m}{2 r}\right)^{4} \delta_{\alpha \beta}+P_{\alpha \beta}
$$

with constants $C_{1}, C_{2}, \ldots, C_{5}$ such that

$$
\left|P_{\alpha \beta}\right| \leqq C_{1} r^{-2}, \quad\left|\partial^{l} P_{\alpha \beta}\right| \leqq C_{l+1} r^{-l-2}, \quad 1 \leqq l \leqq 4,
$$

where $\partial$ denotes partial derivatives with respect to the coordinate system $\left\{y_{\alpha}\right\}$. We set $C_{0}=\max \left(1, m, C_{1}, \ldots, C_{5}\right)$ and write $\mathrm{c}$ for any absolute constant which is independent of the metric $\bar{g}$. Also, we will assume throughout that the mass $m$ is strictly positive.

To compute the curvature of $(N, \bar{g})$, we begin with the special case where $P_{\alpha \beta} \equiv 0$, i.e. where the metric arises from the Schwarzschild metric. 
1.1 Lemma. If $\bar{g}_{\alpha \beta}=g_{\alpha \beta}^{S}=\left(1+\frac{m}{2 r}\right)^{4} \delta_{\alpha \beta}$, then the Ricci curvature $R_{\alpha \beta}^{S}$ of $g_{\alpha \beta}^{S}$ is given by

$$
R_{\alpha \beta}^{S}=\frac{m}{r^{3}} \varphi^{-2}\left(\delta_{\alpha \beta}-3 \frac{y^{\alpha} y^{\beta}}{r^{2}}\right),
$$

where here and in the following $\varphi=\left(1+\frac{m}{2 r}\right)$.

Thus one eigenvalue of the Ricci curvature ( in radial direction ) is given by $-2 m r^{-3} \varphi^{-6}$ and the other two eigenvalues are $m r^{-3} \varphi^{-6}$. The scalar curvature equals zero.

Proof. The metric $g_{\alpha \beta}^{S}$ is conformally Euclidean with conformal factor $\varphi^{4}$. It is wellknown that then

$$
\begin{aligned}
R_{\alpha \beta}^{S}= & -2 \varphi^{-1} \partial_{\alpha} \partial_{\beta} \varphi+6 \varphi^{-2} \partial_{\alpha} \varphi \partial_{\beta} \varphi \\
& -2 \varphi^{-1} \partial_{\alpha} \partial^{\alpha} \varphi \delta_{\alpha \beta}-2 \varphi^{-2}|\partial \varphi|^{2} \delta_{\alpha \beta},
\end{aligned}
$$

and simple computation yields

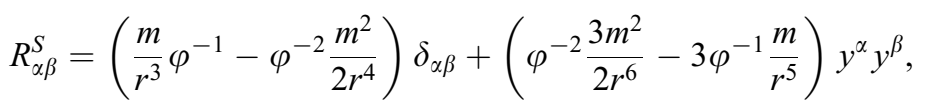

as required.

Near infinity the curvature of the more general metric $\bar{g}$ will be very close to the curvature of $g^{S}$ :

1.2 Lemma. Let $\bar{\nabla}$ and $\bar{R}_{\alpha \beta}$ denote covariant differentiation and Ricci curvature respectively with respect to $\bar{g}$. Then we have

$$
\begin{aligned}
\left|\bar{R}_{\alpha \beta}-R_{\alpha \beta}^{S}\right| & \leqq c \frac{C_{0}}{r^{4}}, \\
\left|\bar{\nabla}_{\gamma} \bar{R}_{\alpha \beta}-\nabla_{\gamma}^{S} R_{\alpha \beta}^{S}\right| & \leqq c \frac{{ }^{C}}{C^{5}}, \\
\left|\bar{\nabla}_{\rho} \bar{\nabla}_{\gamma} \bar{R}_{\alpha \beta}-\nabla_{\rho}^{S} \nabla_{\gamma}^{S} R_{\alpha \beta}^{S}\right| & \leqq c \frac{{ }^{6}}{C^{6}},
\end{aligned}
$$

where $c$ is an absolut constant.

Proof. From the decay assumptions for $P_{\alpha \beta}$ in (1.2) we see that the Christoffel symbols $\bar{\Gamma}_{\beta \delta}^{\alpha}$ of $\bar{g}$ satisfy

$$
\left|\bar{\Gamma}_{\beta \delta}^{\alpha}-\Gamma_{\beta \delta}^{S \alpha}\right| \leqq c{ }^{C_{0}}
$$

with corresponding estimates for the next higher derivatives. This implies the estimates above.

Now let $M$ be a two-dimensional hypersurface in $(N, \bar{g})$. We write $g=\left\{g_{i j}\right\}$ for the induced metric on $M$, Latin indices range from 1 to 2 . Let $v$ be the unit (outward) normal on $M$ and denote covariant differentiation in $M$ by $\nabla$. 
We often work in an orthonormal frame $e_{1}, e_{2}, v$ adapted to $M$, such that the second fundamental form $A=\left\{h_{i j}\right\}$ is given by

$$
h_{i j}=-\left\langle\bar{\nabla}_{e_{i}} e_{j}, v\right\rangle=\left\langle\bar{\nabla}_{e_{i}} v, e_{j}\right\rangle \text {. }
$$

We let $\lambda_{1}$ and $\lambda_{2}$ be the two principle curvatures on $M$ and write

$$
H=g^{i j} h_{i j}=\lambda_{1}+\lambda_{2}, \quad\left|A^{2}\right|=h^{i j} h_{i j}=\lambda_{1}^{2}+\lambda_{2}^{2}
$$

for the mean curvature and the square of the norm of the second fundamental form on $M$ respectively. Furthermore, we let $\AA$ be the traceless second fundamental form such that

$$
\stackrel{\circ}{h}_{i j}=h_{i j}-{ }_{2}^{1} H g_{i j},|\AA|^{2}=|A|^{2}-{ }_{2}^{1} H^{2}={ }_{2}^{1}\left(\lambda_{1}-\lambda_{2}\right)^{2} .
$$

The curvature of $N$ and the second fundamental form of $M$ are related by the equations of Gauß and Codazzi

$$
\begin{aligned}
R_{i j k l} & =\bar{R}_{i j k l}+h_{i k} h_{j l}-h_{i l} h_{j k}, \\
\bar{R}_{3 i j k} & =\nabla_{k} h_{i j}-\nabla_{j} h_{i k},
\end{aligned}
$$

where the index 3 indicates the $v$ direction. Next we state Simons' identity for the Laplacian of the second fundamental form, see e.g. [SSY], and an important consequence of the Codazzi equations, see [Hu1]:

1.3 Lemma. i) The second fundamental form satisfies the identity

$$
\begin{aligned}
\Delta h_{i j}= & \nabla_{i} \nabla_{j} H+H h_{i l} h_{l j}-|A|^{2} h_{i j}+H \bar{R}_{3 i 3 j}-h_{i j} \bar{R}_{3 l 3 l} \\
& +h_{j l} \bar{R}_{l k i k}+h_{i l} \bar{R}_{l k j k}-2 h_{l k} \bar{R}_{l i k j}+\bar{\nabla}_{j} \bar{R}_{3 l i l}+\bar{\nabla}_{l} \bar{R}_{3 i j l}
\end{aligned}
$$

ii) If $w_{i}=\bar{R}_{3 l i l}$ denotes the projection of $\overline{\operatorname{Ric}}(v, \cdot)$ onto $M$, we have for any $\eta>0$ the inequality

$$
|\nabla A|^{2} \geqq\left({ }_{4}^{3}-\eta\right)|\nabla H|^{2}-\left({ }_{4}^{1} \eta^{-1}-1\right)|w|^{2} .
$$

We will also need a formula for the change of the second fundamental form of $M$ after a conformal change of the ambient metric:

1.4 Lemma. Let $M$ be a hypersurface in $(N, \bar{g})$ with principle curvatures $\lambda_{1}$ and $\lambda_{2}$. If $\hat{g}_{\alpha \beta}=\psi^{2} \bar{g}_{\alpha \beta}$ describes a conformal change of the ambient metric, then the eigendirections of the second fundamental form of $M$ remain fixed and the new principle curvatures $\tilde{\lambda}_{1}$ and $\tilde{\lambda}_{2}$ are given by

$$
\tilde{\lambda}_{i}=\psi^{-1} \lambda_{i}+\psi^{-2} \partial_{v} \psi
$$

In particular, the difference between the eigenvalues is conformally invariant:

$$
\left(\tilde{\lambda}_{1}-\tilde{\lambda}_{2}\right)=\psi^{-1}\left(\lambda_{1}-\lambda_{2}\right)
$$


Proof. The relation $\hat{g}_{\alpha \beta}=\psi^{2} \bar{g}_{\alpha \beta}$ implies that the Christoffel symbol $\hat{\Gamma}$ is given by

$$
\hat{\Gamma}_{\beta \gamma}^{\alpha}=\Gamma_{\beta \gamma}^{\alpha}+\psi^{-1}\left(\delta_{\beta}^{\alpha} \partial_{\gamma} \psi+\delta_{\gamma}^{\alpha} \partial_{\beta} \psi-\bar{g}_{\beta \gamma} \bar{g}^{\alpha \rho} \partial_{\rho} \psi\right)
$$

and the result is then an immediate consequence of the definition of the second fundamental form above.

\section{Spherical Hypersurfaces}

The constant mean curvature surfaces constructed in Sect. 3 will be very close to some Euclidean coordinate sphere. In this section we define a class of round surfaces in $N$ and give a detailed description of their geometric properties. In a first proposition we show that exterior surfaces where the traceless part of the second fundamental form is small are close to some Euclidean sphere.

2.1 Proposition. Suppose $M$ is a hypersurface in $(N, \bar{g})$ such that $r(y) \geqq$ ${ }_{10}^{1} \max _{M} r=: r_{1}$ for all $y \in M$ and such that for some constants $K_{1}, K_{2}$

$$
|\AA| \leqq K_{1} r_{1}^{-3}, \quad|\nabla \AA| \leqq K_{2} r_{1}^{-4} .
$$

Then there is an absolute constant $c$ such that the curvature $A^{e}$ of $M$ with respect to the Euclidean metric satisfies

$$
\left|\AA^{e}\right| \leqq c\left(K_{1}+C_{0}\right) r_{1}^{-3}, \quad\left|\nabla^{e} A^{e}\right| \leqq c\left(K_{2}+C_{0}\right) r_{1}^{-4},
$$

provided $r_{1} \geqq c\left(C_{0}+K_{1}\right)$. Moreover, there is a number $r_{0} \in \boldsymbol{R}$ and a vector $\vec{a} \in \boldsymbol{R}^{3}$ such that

$$
\begin{aligned}
\left|\lambda_{i}^{e}-r_{0}^{-1}\right| & \leqq c\left(K_{1}+K_{2}+C_{0}\right) r_{1}^{-3}, \quad i=1,2, \\
\left|(y-\vec{a})-r_{0} v_{e}\right| & \leqq c\left(K_{1}+K_{2}+C_{0}\right) r_{1}^{-1}, \\
\left|v_{e}-r_{0}^{-1}(y-\vec{a})\right| & \leqq c\left(K_{1}+K_{2}+C_{0}\right) r_{1}^{-2} .
\end{aligned}
$$

Here $y$ and $v_{e}$ are the position vector and the unit normal of $M$ in $\boldsymbol{R}^{3}$ respectively.

Proof. Since the Christoffelsymbols of the metric g and the Schwarzschild metric $g^{S}$ are close together, see e.g. Lemma 1.2, the second fundamental form $A^{S}$ of $M$ with respect to the Schwarzschild metric $g^{S}$ satisfies

$$
\left|\AA^{s}\right| \leqq c\left(K_{1}+C_{0}\right) r_{1}^{-3}, \quad\left|\nabla^{s} \AA^{s}\right| \leqq c\left(K_{2}+C_{0}\right) r_{1}^{-4},
$$

provided $r_{1} \geqq c\left(C_{0}+K_{1}\right)$. Since $g^{s}$ is conformally Euclidean, we can now apply Lemma 1.4 to obtain

$$
\left|\AA^{e}\right| \leqq c\left(K_{1}+C_{0}\right) r_{1}^{-3}, \quad\left|\nabla^{e} \AA^{e}\right| \leqq c\left(K_{2}+C_{0}\right) r_{1}^{-4},
$$


provided $r_{1} \geqq c\left(K_{1}+C_{0}\right)$. In the second estimate we also used the fact that the derivative of the conformal factor can be bounded by $c C_{0} r_{1}^{-2}$. In a crucial step we now conclude from Lemma1.3(ii) that in Euclidean space

$$
\left|\nabla^{e} \AA^{e}\right|^{2}=\left|\nabla^{e} A^{e}\right|^{2}-\frac{1}{2}|\nabla H|^{2} \geqq \frac{1}{3}\left|\nabla^{e} A^{e}\right|^{2},
$$

such that here the gradient of the full second fundamental form satisfies $\left|\nabla^{e} A^{e}\right| \leqq c\left(K_{2}+C_{0}\right) r_{1}^{-4}$, proving the first part of the proposition. To find the sphere $S_{r_{0}}(\vec{a})$ close to $M$, first observe that at the point of $M$ where $\max _{M} r=r_{1}$ is attained, we have $\lambda_{i}^{e} \geqq r_{1}^{-1}$. Moreover, since $r_{1} \leqq 10 r$ and in view of the gradient estimate for the curvature there is a number $r_{0}$ such that

$$
\left|\lambda_{i}^{e}-r_{0}^{-1}\right| \leqq c\left(K_{1}+K_{2}+C_{0}\right) r_{1}^{-3}, \quad i=1,2 .
$$

Now consider the Gauß-Weingarten relation

$$
\frac{\partial}{\partial x_{i}} v_{e}=h_{i j}^{e} g^{j k} \frac{\partial y}{\partial x_{k}}
$$

for the exterior Euclidean unit normal and the position vector y. In view of the above estimates we have

$$
\left|\frac{\partial}{\partial x_{i}}\left(v_{e}-r_{0}^{-1} y\right)\right| \leqq c\left(K_{1}+K_{2}+C_{0}\right) r_{1}^{-3}
$$

Clearly we can then choose a vector $\vec{a}$ such that the estimates and the proposition are valid provided $r_{1} \geqq c\left(K_{1}+C_{0}\right)$ and also $r \geqq{ }_{10}^{1} r_{1}$.

For $\sigma \geqq 1$ and $B_{1}, B_{2}, B_{3}$ nonnegative numbers we now define a set $\mathscr{B}_{\sigma}\left(B_{1}, B_{2}, B_{3}\right)$ of round surfaces in $(N, \bar{g})$ by setting

$$
\mathscr{B}_{\sigma}:=\left\{M \subset N\left|\sigma-B_{1} \leqq r \leqq \sigma+B_{1},\right| \AA\left|\leqq B_{2} \sigma^{-3},\right| \nabla \AA \mid \leqq B_{3} \sigma^{-4}\right\} .
$$

If $\sigma$ is sufficiently large compared to $B_{1}, B_{2}, B_{3}$ we may now use the information on the position of the surface in Proposition 2.1 to accurately compute the mean curvature of $M$ in $N$.

2.2 Proposition. Let $M$ be a hypersurface in $B_{\sigma}\left(B_{1}, B_{2}, B_{3}\right)$. Suppose $\sigma \geqq$ $c\left(B_{1}+B_{2}+C_{0}\right)$ is such that all assumptions of Proposition 2.1 are satisfied and let $r_{0}$ and $\vec{a}$ be as in that proposition. Then there is an absolute constant $c$ such that the mean curvature of $M$ satisfies

$$
\left|H-\frac{2}{r_{0}}+\frac{4 m}{r_{0}^{2}}-\frac{6 m\left\langle\vec{a}, v_{e}\right\rangle_{e}}{r_{0}^{3}}\right| \leqq c\left(C_{0}^{2}+B_{2}+B_{3}\right) \sigma^{-3}
$$

provided $\sigma \geqq c\left(B_{1}^{2}+B_{2}+C_{0}\right)$.

Proof. As a first step we compute from Lemma 1.4 the principal curvatures of $S_{r_{0}}(\vec{a})$ with repect to the Schwarzschild metric $g_{\alpha \beta}^{S}=\varphi^{4} \delta_{\alpha \beta}, \varphi=\left(1+{ }_{2 r}^{m}\right)$. We get

$$
\lambda_{i}^{S}=\varphi^{-2} \lambda_{i}^{e}-\frac{m}{r^{3}} \varphi^{-3}\left\langle y, v_{e}\right\rangle_{e},
$$


where as before $y$ is the position vector, $r=r(y)$ is the Euclidean distance and $\langle,\rangle_{e}$ is the Euclidean inner product. In view of the estimates in Proposition 2.1 we have

$$
\left|\lambda_{i}^{S}-\frac{1}{r_{0}} \varphi^{-2}+\frac{m r_{0}}{r^{3}} \varphi^{-3}+\frac{m}{r^{3}} \varphi^{-3}\langle\vec{a}, v\rangle_{e}\right| \leqq c\left(B_{2}+B_{3}+C_{0}\right) \sigma^{-3} .
$$

Observe that

$$
\left|\lambda_{i}^{S}-\lambda_{i}\right|=\frac{1}{r_{0}}\left|\varphi^{-2}-\left(1-\frac{m}{r}\right)\right|+\frac{1}{r}\left|\varphi^{-3}-\left(1-\frac{3 m}{2 r}\right)\right| \leqq c C_{0} \sigma^{-3},
$$

such that we get the estimate

$$
\left|\lambda_{i}-\frac{1}{r_{0}}+\frac{m}{r r_{0}}+\frac{m r_{0}}{r^{3}}+\frac{m\langle\vec{a}, v\rangle_{e}}{r^{3}}\right| \leqq c C_{0} \sigma^{-3}
$$

when $\sigma \geqq c B_{1}$. Now notice that

$$
\left|r-\left(r_{0}+\langle\vec{a}, v\rangle_{e}\right)\right| \leqq \frac{|\vec{a}|^{2}}{2 r_{0}} .
$$

Then we obtain for sufficiently large $\sigma \geqq c B_{1}^{2}$ that

$$
\left|\lambda_{i}-\frac{1}{r_{0}}+\frac{2 m}{r_{0}^{2}}-\frac{3 m\langle\vec{a}, v\rangle_{e}}{r_{0}^{3}}\right| \leqq c\left(C_{0}^{2}+B_{2}+B_{3}\right) \sigma^{-3},
$$

as required.

\section{Existence of constant mean curvature surfaces}

We want to show here that for sufficiently large $\sigma$, related to each coordinate sphere $S_{\sigma}(0)$ there is a constant mean curvature surface which is round in the sense of Proposition 2.1. To accomplish this we take $S_{\sigma}(0)$ as an initial surface and evolve it in direction of its unit normal in $N$ with speed given by $(H-h)$, where $h=\oint h d \mu$ is the mean value of the mean curvature. This flow keeps the volume of the evolving surfaces with respect to some fixed reference slice constant and decreases area at the same time. It was first studied for hypersurfaces in $\boldsymbol{R}^{n+1}$ in [Hu1], and later for surfaces in Lorentzian ambient manifolds in [EH1].

To be precise let $S_{\sigma}(0)$ in $(N, g)$ be given by a map

$$
F_{0}^{\sigma}: S^{2} \rightarrow N, \quad F_{0}^{\sigma}\left(S^{2}\right)=S_{\sigma}(0) .
$$

Then we want to find a one-parameter family of maps $F_{t}^{\sigma}=F^{\sigma}(\cdot, t)$ such that the initial value problem

$$
\begin{aligned}
\frac{d}{d t} F^{\sigma}(p, t) & =(h-H) v(p, t), \quad t \geqq 0, p \in S^{2} \\
F^{\sigma}(0) & =F_{0}^{\sigma}
\end{aligned}
$$


is satisfied. Here $H(p, t), v(p, t)$ are the mean curvature and the exterior unit normal at $F^{\sigma}(p, t)$ of the surface $M_{t}=F_{t}^{\sigma}\left(S^{2}\right)$. We will omit the superscript $\sigma$ in the following whenever the meaning is clear from the context.

3.1 Theorem. If the metric $g$ of $N$ is as described in section 1 and if $m>0$, then there is $\sigma_{0}$ depending only on $C_{0}$ and $m$ such that for all $\sigma \geqq \sigma_{0}$ the initial value problem $(\sigma)$ has a unique smooth solution for all times $t \geqq 0$. As $t \rightarrow \infty$, the surfaces converge exponentially fast to a hypersurface $M^{\sigma}$ of constant mean curvature $H_{\sigma}$. There are constants $C_{1}$ and $C_{2}$ depending only on $C_{0}$ and $m$ but not on $\sigma$ such that $|r-\sigma| \leqq C_{1}$ and also $\left|H_{\sigma}-{ }_{\sigma}^{2}+{ }_{\sigma^{2}}\right| \leqq C_{2} \sigma^{-3}$.

Problem $(\sigma)$ is a quasilinear parabolic system and it is well known that a shorttime solution exists:

3.2 Lemma. If the initial data $F_{0}$ are smooth, then the initial value problem $(\sigma)$ has a unique smooth solution $M_{t}$ on some time interval $0 \leqq t<\varepsilon$.

For general initial data solutions to $(\sigma)$ may develop singularities, e.g. neckpinching. For the proof of Theorem 3.1 we will show that $M_{t}$ remains round during the evolution, the first goal is

3.3 Theorem. There are constants $\sigma_{0}, B_{1}, B_{2}, B_{3}$ depending only on $m$ and $C_{0}$ such that for all $\sigma \geqq \sigma_{0}$ the solution of $(\sigma)$ remains in $B_{\sigma}\left(B_{1}, B_{2}, B_{3}\right)$ as long as it exists.

We will in the following always assume that $\sigma, B_{1}, B_{2}, B_{3}$ are chosen such that $M_{0}$ is strictly inside $B_{\sigma}\left(B_{1}, B_{2}, B_{3}\right)$. In the next two propositions we control the position of $M_{t}$ during the evolution by estimates which are independent of $B_{1}$.

3.4 Proposition. Suppose $M_{t}$ is a smooth solution of the initial value problem $(\sigma)$ which is contained in $B_{\sigma}\left(B_{1}, B_{2}, B_{3}\right)$ for all $t \in[0, T]$. Suppose that $\sigma \geqq$ $c\left(C_{0}+B_{1}+B_{2}\right)$ is such that Proposition 2.1 applies and let $r_{0}(t)$ be as in that result. Then there is an absolute constant $c$ such that

$$
\left|r_{0}(t)-\sigma\right| \leqq c\left(C_{0}+B_{2}+B_{3}\right)
$$

holds uniformly in $[0, T]$, provided $\sigma \geqq c\left(C_{0}+B_{1}+B_{2}\right)$.

Proof. At time $t=0$ we have $r_{0}=\sigma$ and we only have to prove (2.1) under the assumption that $\sigma / 2 \leqq r_{0}(t) \leqq 2 \sigma$. To see that $r_{0}(t)$ doesn't change much, observe that in view of equation $(\sigma)$ the volume $V_{N}$ of the shell $W$ between $M_{t}$ and a fixed reference surface in $N$, e.g. the surface $S_{\sigma / 2}(0)$ in $N$ is constant and equal to $\pi \sigma^{3}$ up to terms of order $\sigma^{2}$. If $\sigma \geqq c\left(C_{0}+B_{1}+B_{2}\right)$ is such that Proposition 2.1 is applicable and also $r \geqq{ }_{4}^{3} \sigma$ everywhere then we get for the Euclidean volume $V_{e}$ of $W$ that

$$
\left|V_{e}-V_{N}\right| \leqq \int_{W} 1-\sqrt{ } \operatorname{det} g d x \leqq c C_{0} \sigma^{2}
$$


But from the estimates in Proposition 2.1 we get that $V_{e}$ equals ${ }_{3}^{4} \pi r_{0}^{3}-{ }_{3}^{1} \pi \sigma^{3}$ up to terms of order $\sigma^{2}$, such that

$$
\left|{ }_{3}^{4} \pi \sigma^{3}-{ }_{3}^{4} \pi r_{0}^{3}\right| \leqq c\left(C_{0}+B_{2}+B_{3}\right) \sigma^{2},
$$

which implies the desired estimate for $\sigma \geqq c\left(C_{0}+B_{1}+B_{2}\right)$.

3.5 Proposition. Suppose that the solution $M_{t}$ of $(\sigma)$ is contained in $B_{\sigma}\left(B_{1}, B_{2}\right.$, $\left.B_{3}\right)$ for $t \in[0, T]$. Then there is an absolute constant $c$ such that

$$
\max _{M_{t}} r \leqq \sigma+c\left(m^{-1}+1\right)\left(C_{0}^{2}+B_{2}+B_{3}\right)
$$

holds for all $t \in[0, T]$ provided $\sigma \geqq c\left(C_{0}^{2}+B_{1}^{2}+B_{2}+B_{3}\right)$.

Proof. Suppose that $\sigma \geqq c\left(C_{0}+B_{1}^{2}+B_{2}\right)$ is such that the assumptions of Propositions 2.1 and 2.2 hold, and let $\vec{a}$ and $r_{0}$ be the approximate centre and the approximate radius of $M_{t}$ established there. Now let $D>0$ and assume that the condition $\max _{M_{t}} r<\sigma+D$ is violated for the first time at $\left(y_{0}, t_{0}\right), t_{0}>0$. At that point $r$ attains its maximum and thus $\left\langle y_{0}, v_{e}\right\rangle_{e}=r$. Moreover, since $t_{0}$ is the first time where $r$ reaches $\sigma+D$, at $\left(y_{0}, t_{0}\right)$ the speed of $M_{t}$ with respect to the Euclidean unit normal is non-negative, i.e. $(h-H)\left\langle v, v_{e}\right\rangle_{e} \geqq 0$. From the estimates in Proposition 2.1 it follows that $\left\langle v, v_{e}\right\rangle_{e} \geqq 1 / 2$ and also $\left\langle\vec{a}, v_{e}\right\rangle_{e} \geqq \frac{1}{2}|\vec{a}|$ at this point provided $\sigma \geqq c\left(C_{0}+B_{2}+B_{3}\right)$. So we have $H \leqq h$ at $\left(y_{0}, t_{0}\right)$, but on the other hand we get from Proposition 2.2 that there

$$
\begin{aligned}
H-h & \geqq \frac{3 m|\vec{a}|}{r_{0}^{3}}-\frac{6 m}{r_{0}^{3}} \oint_{M_{t_{0}}}\left\langle\vec{a}, v_{e}\right\rangle_{e} d \mu_{t}-c\left(C_{0}^{2}+B_{2}+B_{3}\right) \sigma^{-3} \\
& \geqq\left(2 m|\vec{a}|-c\left(C_{0}^{2}+B_{2}+B_{3}\right)\right) \sigma^{-3},
\end{aligned}
$$

provided $\sigma \geqq c\left(C_{0}^{2}+B_{1}^{2}+B_{2}+B_{3}\right)$. Since at $\left(y_{0}, t_{0}\right)$ the radius satisfies $r=\sigma+D$ and in view of Proposition 2.1 $\left|r-\left(r_{0}+|\vec{a}|\right)\right| \leqq c\left(C_{0}+B_{2}+B_{3}\right) \sigma^{-1}$, we see from Proposition 3.4 that

$$
|\vec{a}| \geqq D-c\left(C_{0}+B_{2}+B_{3}\right) \quad \text { at }\left(y_{0}, t_{0}\right) .
$$

Thus (2.2) yields a contradiction if $D$ is larger than $c\left(m^{-1}+1\right)\left(C_{0}^{2}+B_{2}+B_{3}\right)$, completing the proof.

To obtain in the next step a priori estimates for $|\AA|^{2}$, we need evolution equations for the metric and the second fundamental form on $M_{t}$. Computing as in [Hu1] and [Hu2] we obtain from $(\sigma)$ :

3.6 Lemma. We have the evolution equations

$$
\begin{aligned}
\frac{d}{d t} g_{i j} & =2(h-H) h_{i j}, \\
\frac{d}{d t} v & =\nabla H,
\end{aligned}
$$


(iii)

$$
\begin{aligned}
\frac{d}{d t} \mu & =H(h-H) \mu \\
\frac{d}{d t} h_{i j} & =\nabla_{i} \nabla_{j} H+(h-H) h_{i l} h_{l j}-(h-H) \bar{R}_{3 i 3 j},
\end{aligned}
$$

where $\mu$ is the measure on $M_{t}$.

Using Simons' identity, Lemma 1.3(i), we can then easily obtain the following additional equations, computing exactly as in [Hul].

3.7 Lemma. The second fundamental form satisfies the evolution equations

$$
\begin{aligned}
\frac{d}{d t} h_{i j}= & \Delta h_{i j}-2 H h_{i l} h_{l j}+h h_{i l} h_{l j}+|A|^{2} h_{i j}-h \bar{R}_{3 i 3 j}+h_{i j} \bar{R}_{3 l 3 l} \\
& -h_{j l} \bar{R}_{l m i m}-h_{i l} \bar{R}_{l m j m}+2 h_{l m} \bar{R}_{l i m j}-\bar{\nabla}_{j} \bar{R}_{3 l i l}-\bar{\nabla}_{l} \bar{R}_{3 i j l}, \\
\frac{d}{d t} H & =\Delta H+(H-h)\left(|A|^{2}+\bar{R} i c(v, v)\right), \\
{ }_{d}^{d}|A|^{2}= & \Delta|A|^{2}-2|\nabla A|^{2}+2|A|^{4}-2 h \operatorname{tr}\left(A^{3}\right)-2 h h_{i j} \bar{R}_{3 i 3 j} \\
d t & +2|A|^{2} \bar{R} i c(v, v)-4\left(h_{i j} h_{j l} \bar{R}_{l m i m}-h_{i j} h_{l m} \bar{R}_{l i m j}\right) \\
& -2 h_{i j}\left(\bar{\nabla}_{j} \bar{R}_{3 l i l}-\bar{\nabla}_{l} \bar{R}_{3 i j l}\right) .
\end{aligned}
$$

We also need some technical estimates for the expressions in the last Lemma.

3.8 Lemma. Let $M_{t}$ be a smooth solution of $(\sigma)$ contained in $B_{\sigma}\left(B_{1}, B_{2}, B_{3}\right)$ for $t \in[0, T]$. Then there is an absolute constant $c$ such that for $\sigma \geqq c\left(C_{0}^{2}+\right.$ $\left.B_{1}^{2}+B_{2}\right)$

$$
\begin{aligned}
\left|\lambda_{i}-\frac{1}{\sigma}\right|+|H-h| & \leqq c\left(C_{0}^{2}+B_{2}+B_{3}\right) \sigma^{-2}, \\
\frac{2 h}{H^{3}}\left(|A|^{4}-H \operatorname{tr}\left(A^{3}\right)\right) & \leqq-\frac{1}{2}|\AA|^{2}, \\
\frac{h}{H^{2}}\left|\stackrel{\circ}{h i j}_{i j 3}\right| & \leqq c C_{0}|\AA| \sigma^{-3}, \\
\left|h_{i j} h_{j l} \bar{R}_{l m i m}-h_{i j} h_{l m} \bar{R}_{l i m j}\right| & \leqq c C_{0}|\AA|^{2} \sigma^{-3}, \\
\left|h_{i j}\left(\bar{\nabla}_{j} \bar{R}_{3 l i l}+\bar{\nabla}_{l} \bar{R}_{3 i j l}\right)\right| & \leqq c C_{0}|\AA| \sigma^{-5} .
\end{aligned}
$$

Proof. The first inequality is an easy consequence of Propositions 2.2 and 3.4. To obtain the second inequality observe that

$$
|A|^{4}-H \operatorname{tr}\left(A^{3}\right)=-\lambda_{1} \lambda_{2}\left(\lambda_{1}-\lambda_{2}\right)^{2}=-2 \lambda_{1} \lambda_{2}|\AA|^{2},
$$

which implies the desired estimate in view of (i). Now recall that the Riemann curvature tensor on a three-dimensional manifold can be expressed by the Ricci curvature:

$$
\bar{R}_{\alpha \beta \gamma \delta}=\left(\bar{R}_{\alpha \gamma} g_{\beta \delta}-\bar{R}_{\alpha \delta} g_{\beta \gamma}-\bar{R}_{\beta \gamma} g_{\alpha \delta}+\bar{R}_{\beta \delta} g_{\alpha \gamma}\right)-{ }_{2}^{1} \bar{R}\left(g_{\alpha \gamma} g_{\beta \delta}-g_{\alpha \delta} g_{\beta \gamma}\right) .
$$


It follows that

$$
\stackrel{\circ}{h}_{i j} \bar{R}_{i 3 j 3}=\frac{1}{2}\left(\lambda_{1}-\lambda_{2}\right)\left(\bar{R}_{11}-\bar{R}_{22}\right),
$$

but from Lemmata 1.1 and 1.2, using also the estimates in Proposition 2.1, we get $\left|\bar{R}_{11}-\bar{R}_{22}\right| \leqq c C_{0} \sigma^{-4}$ for $\sigma \geqq c\left(C_{0}+B_{1}+B_{2}+B_{3}\right)$ as desired. The fourth estimate follows from the identity

$$
h_{i j} h_{j l} \bar{R}_{l m i m}-h_{i j} h_{l m} \bar{R}_{l i m j}=\left(\lambda_{1}-\lambda_{2}\right)^{2} \bar{R}_{1212},
$$

formula (3.3) above and Lemma 1.1. Finally, using (3.3) again we find

$$
h_{i j}\left(\bar{\nabla}_{j} \bar{R}_{3 l i l}+\bar{\nabla} \bar{R}_{3 i j l}\right)=\frac{1}{2}\left(\lambda_{1}-\lambda_{2}\right)\left(\bar{\nabla}_{1} \bar{R}_{31}-\bar{\nabla}_{2} \bar{R}_{32}\right),
$$

and the conclusion follows as in (iii) from Lemmata 1.1 and 1.2 and from Proposition 2.1.

We are now ready to prove an a priori estimate for the difference between the principal curvatures.

3.9 Proposition. Suppose that the solution $M_{t}$ of $(\sigma)$ is contained in $B_{\sigma}\left(B_{1}, B_{2}\right.$, $\left.B_{3}\right)$ for $t \in[0, T]$. Then there is an absolute constant $c$ such that for $\sigma \geqq$ $c\left(C_{0}^{2}+B_{1}^{2}+B_{2}+B_{3}\right)$ we have the estimate

$$
|\AA|^{2} \leqq c C_{0}^{2} \sigma^{-6}
$$

everywhere in $[0, T]$.

Proof. Consider the function $f=|\AA|^{2} / H^{2}$. Computing as in [Hu1] we obtain from Lemma 3.7(ii),(iii) that

$$
\begin{aligned}
\frac{d}{d t} f= & \Delta f+\frac{2}{H}\left\langle\nabla_{l} H, \nabla_{l} f\right\rangle-\frac{2}{H^{4}}\left|\nabla_{i} H h_{k l}-\nabla_{i} h_{k l} H\right|^{2}+\frac{2 h}{H^{3}}\left(|A|^{4}-H \operatorname{tr}\left(A^{3}\right)\right) \\
& +\frac{2 h}{H} f \bar{R} i c(v, v)-\frac{2 h}{H^{2}} \stackrel{\circ}{i j}_{i j} \bar{R}_{i 3 j 3}-\frac{1}{H^{2}}\left[4\left(h_{i j} h_{j l} \bar{R}_{l m i m}-h_{i j} h_{l m} \bar{R}_{l i m j}\right)\right. \\
& \left.+2 h_{i j}\left(\bar{\nabla}_{j} \bar{R}_{3 l i l}+\bar{\nabla}_{l} \bar{R}_{3 i j l}\right)\right] .
\end{aligned}
$$

Using now all the estimates in Lemma 3.8 and the fact that $\bar{R} i c(v, v)$ is negative for $\sigma \geqq c\left(C_{0}+B_{2}+B_{3}\right)$ by Lemmata 1.1, 1.2 and Proposition 2.1, we conclude that

$$
\frac{d}{d t} f \leqq \Delta f+\frac{2}{H}\left\langle\nabla_{l} H, \nabla_{l} f\right\rangle-\frac{1}{2}|\AA|^{2}+c C_{0}|\AA| \sigma^{-3},
$$

provided $\sigma \geqq c\left(C_{0}^{2}+B_{1}^{2}+B_{2}+B_{3}\right)$ is so large that Propositions 2.1, 2.2, 3.4, 3.5 and Lemma 3.8 all apply. Now suppose that $f$ reaches a value $D \sigma^{-4}$ for the first time at $\left(y_{0}, t_{0}\right)$, where $D$ is some positive number larger than the initial values of $f \sigma^{4}$. Then at $\left(y_{0}, t_{0}\right)$ we have $(d / d t) f \geqq 0, \Delta f \leqq 0$ and $\nabla f=0$ such that

$$
0 \leqq-{ }_{2}^{1}|\AA|^{2}+c C_{0}|\AA| \sigma^{-3} \quad \text { at }\left(y_{0}, t_{0}\right) \text {. }
$$


Since at that point $f=|\AA|^{2} / H^{2}=D \sigma^{-4}$ and since $\sigma^{-1} \leqq H \leqq 3 \sigma^{-1}$ we get

$$
0 \leqq-{ }_{2}^{1} D \sigma^{-6}+c C_{0} D^{1 / 2} \sigma^{-6},
$$

which is a contradiction for $D \geqq c C_{0}^{2}$. Hence $f \leqq c C_{0}^{2} \sigma^{-4}$, completing the proof of the proposition.

In order to prove an a priori estimate for the gradient of the curvature, we introduce the following notation: We write $A * B$ for any linear combination of contractions of $A$ and $B$ with the metric $g_{i j}$. Then the evolution equation for the curvature gradient has the following form.

3.10 Lemma. The gradient of the second fundamental form satisfies

$$
\begin{aligned}
& \frac{d}{d t}|\nabla \AA|^{2}=\Delta|\AA|^{2}-2\left|\nabla^{2} \AA\right|^{2}+\nabla A * \nabla \AA * A * \AA+\nabla \AA * \AA * A * \bar{R} i c \\
& +\nabla \AA * \nabla \AA * A * A+\nabla \AA * \nabla \AA * \bar{R} i c+\nabla \AA * \AA * \bar{\nabla} \bar{R} i c \\
& -2 h \nabla_{k} \stackrel{\circ}{h}_{i j} \nabla_{k}\left(\bar{R}_{3 i 3 j}-{ }_{2}^{1} \bar{R} i c(v, v) g_{i j}\right)-2 \nabla_{k} \stackrel{\circ}{h}_{i j} \nabla_{k}\left(\bar{\nabla}_{j} \bar{R}_{3 l i l}\right. \\
& \left.+\bar{\nabla}_{l} \bar{R}_{3 i j l}\right) \text {, }
\end{aligned}
$$

where for simplicity we also wrote A for the tensor $h g_{i j}$.

Proof. Since $(d / d t) g_{i j}=2(h-H) h_{i j}$, the time derivative of the Christoffel symbols is of the form $A * \nabla A$ and thus

$$
\begin{aligned}
& \frac{d}{d t}|\nabla \stackrel{\circ}{A}|^{2}=2\left(\frac{d}{d t} \nabla_{k} \stackrel{\circ}{h_{i j}}\right) \nabla_{k} \stackrel{\circ}{h}_{i j}
\end{aligned}
$$

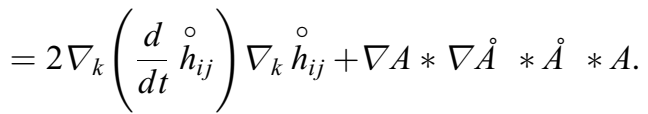

From Lemma 3.7(i),(ii) we compute after some long but easy calculations

$$
\begin{aligned}
\frac{d}{d t} \stackrel{\circ}{h}_{i j}= & \Delta \stackrel{\circ}{h}_{i j}+(h-2 H) \stackrel{\circ}{h} \stackrel{\circ}{h}_{l j}-{ }_{2}^{1} H^{2} \stackrel{\circ}{h}_{i j}+|\stackrel{\circ}{\mid}|^{2}\left(\stackrel{\circ}{h}_{i j}+{ }_{2}^{1} h g_{i j}\right) \\
& +\stackrel{\circ}{h}_{i j} \bar{R} i c(v, v)-h\left(\bar{R}_{3 i 3 j}-{ }_{2} \bar{R}_{i} i c(v, v)\right) g_{i j} \\
& -\stackrel{\circ}{h}_{j l} \bar{R}_{l m i m}-\stackrel{\circ}{h}_{i l} \bar{R}_{l m j m}+2 \stackrel{\circ}{h}_{l m} \bar{R}_{l i m j}-\bar{\nabla}_{j} \bar{R}_{3 l i l}-\bar{\nabla}_{l} \bar{R}_{3 i j l},
\end{aligned}
$$

such that

$$
\begin{aligned}
\frac{d}{d t} \stackrel{\circ}{h}_{i j}= & \Delta \stackrel{\circ}{h}_{i j}+\AA * A * A+\AA * \bar{R} i c \\
& -h\left(\bar{R}_{3 i 3 j}-{ }_{2}^{1} \bar{R} i c(v, v)\right) g_{i j}-\bar{\nabla}_{j} \bar{R}_{3 l i l}-\bar{\nabla}_{l} \bar{R}_{3 i j l} .
\end{aligned}
$$


Hence we obtain

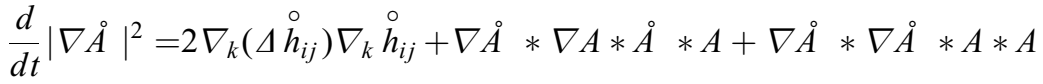

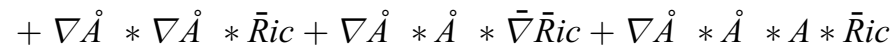

$$
\begin{aligned}
& -2 h \nabla_{k} \stackrel{\circ}{h}_{i j} \nabla_{k}\left(\bar{R}_{3 i 3 j}-{ }_{2}^{1} \bar{R} i c(v, v)\right) g_{i j}-2 \nabla_{k} \stackrel{\circ}{h}_{i j} \nabla_{k}\left(\bar{\nabla}_{j} \bar{R}_{3 l i l}\right. \\
& \left.+\bar{\nabla}_{l} \bar{R}_{3 i j l}\right) \text {. }
\end{aligned}
$$

Now observe that after changing the order of derivatives we get

$$
\begin{aligned}
2 \nabla_{k}\left(\Delta \stackrel{\circ}{h}_{i j}\right) \nabla_{k} \stackrel{\circ}{h}_{i j}= & 2 \Delta\left(\nabla_{k} \stackrel{\circ}{h i j}\right) \nabla_{k} \stackrel{\circ}{h}_{i j} \\
= & \Delta|\AA|^{2}-2\left|\nabla^{2} \AA\right|^{2}+\nabla \AA * \nabla \AA * \bar{R} i c \\
& +\nabla \AA * \AA * \bar{\nabla} \bar{R} i c+\nabla \AA * \AA * A * \bar{R} i c,
\end{aligned}
$$

which implies the result.

3.11 Corollary. If $M_{t}$ is a solution of $(\sigma)$ contained in $B_{\sigma}\left(B_{1}, B_{2}, B_{3}\right)$, then there is an absolute constant $c$ such that

$$
\frac{d}{d t}|\nabla \AA|^{2} \leqq \Delta|\nabla \AA|^{2}+c|\nabla \AA|^{2} \sigma^{-2}+c C_{0}|\nabla \AA| \sigma^{-6}
$$

provided $\sigma \geqq c\left(C_{0}^{2}+B_{1}^{2}+B_{2}\right)$.

Proof. First observe that in view of Lemma 1.3(ii)

$$
\begin{aligned}
|\nabla A|^{2}=|\nabla \AA|^{2}+\frac{1}{2}|\nabla H|^{2} & \leqq 5|\nabla \AA|^{2}+4 \sum_{i}\left|\bar{R} i c\left(v, e_{i}\right)\right|^{2} \\
& \leqq 5|\nabla \AA|^{2}+c\left(C_{0}^{2}+B_{1}^{2}\right) \sigma^{-8},
\end{aligned}
$$

where we computed $\bar{R} i c(v, v)$ as in the proof of Proposition 2.3. Assuming then that $\sigma \geqq c\left(C_{0}^{2}+B_{1}^{2}+B_{2}+B_{3}\right)$ is so large that Proposition 3.8 applies, we get

$$
\begin{aligned}
|\nabla A * \nabla \AA * A * \AA| & \leqq c B_{2}\left(C_{0}+B_{1}\right)|\nabla \AA| \sigma^{-8}, \\
|\nabla \AA * \AA * A * \bar{R} i c| & \leqq c C_{0} B_{2}|\nabla \AA| \sigma^{-7} \\
|\nabla \AA * \nabla \AA * A * A| & \leqq c|\nabla \AA|^{2} \sigma^{-2} \\
|\nabla \AA * \nabla \AA * \bar{R} i c| & \leqq c C_{0}|\nabla \AA|^{2} \sigma^{-3} \\
|\nabla \AA * \AA * \bar{\nabla} i c| & \leqq c C_{0} B_{2}|\AA| \sigma^{-7} .
\end{aligned}
$$

Furthermore, we can argue exactly as in the proof of Proposition 3.8(iii) and $3.8(\mathrm{v})$ to obtain the estimates

$$
\left|2 h \nabla_{k} \stackrel{\circ}{h}_{i j} \nabla_{k}\left(\bar{R}_{3 i 3 j}-{ }_{2}^{1} \bar{R} i c(v, v) g_{i j}\right)\right| \leqq c C_{0}|\nabla \AA| \sigma^{-6}
$$

and

$$
\left|2 \nabla_{k} \stackrel{\circ}{h}_{i j} \nabla_{k}\left(\bar{\nabla}_{j} \bar{R}_{3 l i l}+\bar{\nabla} R_{3 i j l}\right)\right| \leqq c C_{0}|\nabla \AA| \sigma^{-6} .
$$


This implies the estimate of the Corollary for $\sigma \geqq c\left(C_{0}^{2}+B_{1}^{2}+B_{2}+B_{3}\right)$.

We are now ready to prove the a priori estimate for the gradient of the curvature.

3.12 Proposition. Suppose that the solution $M_{t}$ of $(\sigma)$ is contained in $B_{\sigma}\left(B_{1}, B_{2}\right.$, $\left.B_{3}\right)$ for $t \in[0, T]$. Then there is an absolute constant $c$ such that for $\sigma \geqq c\left(C_{0}^{2}+B_{1}^{2}+B_{2}+B_{3}\right)$ the estimate

$$
|\nabla \AA|^{2} \leqq c C_{0}^{2} \sigma^{-8}
$$

holds everywhere in $[0, T]$.

Proof. First observe that by Lemma 3.7 (iii) and (ii), as well as by Lemma (3.8) (iii), (iv) and (v) we have the evolution equation

$$
\begin{aligned}
\frac{d}{d t}|\AA|^{2}= & \Delta|\AA|^{2}-2|\nabla \AA|^{2}+\left(\lambda_{1}-\lambda_{2}\right)^{2}\left(\lambda_{1}^{2}+\lambda_{2}^{2}-\lambda_{1} h-\lambda_{2} h\right) \\
& +2|\AA|^{2} \bar{R} i c(v, v)-2 h \stackrel{\circ}{h}_{i j} \bar{R}_{3 i 3 j}-4\left(h_{i j} h_{j l} \bar{R}_{l m i m}-h_{i j} h_{l m} \bar{R}_{l i m j}\right) \\
& -2 h_{i j}\left(\bar{\nabla}_{j} \bar{R}_{3 l i l}-\bar{\nabla}_{l} \bar{R}_{3 i j l}\right) \leqq \Delta|\AA|^{2}-2|\nabla \AA|^{2}+c C_{0}|\AA| \sigma^{-5} .
\end{aligned}
$$

The last inequality follows for $\sigma \geqq c\left(C_{0}^{2}+B_{1}^{2}+B_{2}\right)$ from Lemma 3.8. Now let $C_{1}$ be sufficiently large compared to the absolute constant $c_{1}$ in Corollary 3.11. Then

$$
\begin{aligned}
\frac{d}{d t}\left(|\nabla \AA|^{2}+C_{1}|\AA|^{2} \sigma^{-2}\right) \leqq & \triangle\left(|\nabla \AA|^{2}+C_{1}|\AA|^{2} \sigma^{-2}\right) \\
& -c_{1}|\nabla \AA|^{2} \sigma^{-2}+c C_{0}|\nabla \AA| \sigma^{-6}+c C_{0}^{2} \sigma^{-10}
\end{aligned}
$$

where we also used Proposition 3.9 for $\sigma \geqq c\left(C_{0}^{2}+B_{1}^{2}+B_{2}+B_{3}\right)$. Using the parabolic maximum principle as before the desired estimate now follows from Proposition 3.9.

Summarising our a priori estimates in Propositions 3.5, 3.9 and 3.12, we have shown that there is a uniform absolute constant $c_{2}$ such that

$$
\begin{aligned}
\max _{M_{t}}|r-\sigma| & \leqq c_{2}\left(m^{-1}+1\right)\left(C_{0}^{2}+B_{2}+B_{3}\right), \\
\max _{M_{t}}|\AA| & \leqq c_{2} C_{0} \sigma^{-3}, \\
\max _{M_{t}}|\nabla \AA| & \leqq c_{2} C_{0} \sigma^{-4},
\end{aligned}
$$

provided that $\sigma \geqq c_{2}\left(C_{0}^{2}+B_{1}^{2}+B_{2}+B_{3}\right)$. Thus we may first choose $B_{2}>$ $c_{2} C_{0}, B_{3}>c_{2} C_{0}$, then choose $B_{1}$ larger than $c_{2}\left(m^{-1}+1\right)\left(C_{0}^{2}+B_{2}+B_{3}\right)$ and finally $\sigma_{0} \geqq c_{2}\left(C_{0}^{2}+B_{1}^{2}+B_{2}+B_{3}\right)$ to ensure that the solution $M_{t}$ of $(\sigma)$ remains strictly inside $B_{\sigma}\left(B_{1}, B_{2}, B_{3}\right), \sigma \geqq \sigma_{0}$, as long as it exists. This completes the proof of Theorem 3.3.

Once we have obtained Theorem 3.3, higher derivative estimates and longtime existence are easily obtained, see e.g. [Hu1] and [Hu2]. We have only to 
show that the solution surfaces $M_{t}$ converge exponentially fast to a constant mean curvature limiting surface $M_{\infty}$. For that purpose we need the following lower bound for the first eigenvalue of the Laplace-Beltrami operator on $M_{t}$.

3.13 Lemma. Suppose $M$ is a hypersurface of $N$ in $B_{\sigma}\left(B_{1}, B_{2}, B_{3}\right)$. Then the lowest eigenvalue $\mu_{\text {Lap }}$ of the negative of the Laplace-Beltrami operator on $M$ satisfies the inequality

$$
\mu_{\text {Lap }} \geqq \frac{2}{\sigma^{2}}-\frac{4 m}{\sigma^{3}}-c C_{0} B_{1} \sigma^{-4},
$$

provided $\sigma \geqq \sigma_{0} \geqq c\left(C_{0}^{2}+B_{1}^{2}+B_{2}+B_{3}\right)$.

Proof. On a two-dimensional surface the Laplace-Beltrami operator has its lowest eigenvalue bounded by

$$
\mu_{\text {Lap }} \geqq 2 K,
$$

where $K$ is a lower bound for the Gauß curvature on $M$. But by Gauß' equation we have

$$
K \geqq \bar{\sigma}_{12}+\lambda_{1} \lambda_{2}
$$

where $\sigma_{12}$ is the sectional curvature of $N$ in tangential direction and the assertion follows from Propositions 2.1, 2.2 and also Lemmata 1.1 and 1.2.

To prove now exponential convergence to a constant mean curvature surface, we compute from Lemma 3.6(iii) and from Lemma 3.7(ii) that

$$
\begin{array}{rl}
\frac{d}{d t} \int_{M_{t}}(H-h)^{2} & d \mu=\int_{M_{t}} 2(H-h) \frac{d}{d t}(H-h) d \mu-\int_{M_{t}}(H-h)^{3} H d \mu \\
= & 2 \int_{M_{t}}(H-h)\left(\Delta H+(H-h)\left(|A|^{2}+\bar{R} i c(v, v)\right)\right) d \mu \\
& -\int_{M_{t}}(H-h)^{3} H d \mu,
\end{array}
$$

where we also used that $\int_{M_{t}} H-h d \mu=0$. After integration by parts we obtain from Lemma 3.13

$$
\begin{aligned}
& \frac{d}{d t_{M_{t}}}(H-h)^{2} d \mu \leqq-\left(\frac{4}{\sigma^{2}}-\frac{8 m}{\sigma^{3}}-c C_{0} B_{1} \sigma^{-4}\right) \int_{M_{t}}(H-h)^{2} d \mu \\
&+2 \int_{M_{t}}(H-h)^{2}\left(|A|^{2}+\bar{R} i c(v, v)\right) d \mu-\int_{M_{t}}(H-h)^{3} H d \mu .
\end{aligned}
$$

From Lemmata 1.1, 1.2 and Propositions 2.1 and 2.2 we know

$$
|A|^{2}+\bar{R} i c(v, v) \leqq \frac{2}{\sigma^{2}}-\frac{10 m}{\sigma^{3}}+c C_{0} B_{1} \sigma^{-4},
$$

such that for $\sigma \geqq \sigma_{0} \geqq c C_{0} B_{1}$ sufficiently large, some $\varepsilon>0$ small

$$
\frac{d}{d t} \int_{M_{t}}(H-h)^{2} d \mu \leqq-\frac{(12-\varepsilon) m}{\sigma^{3}} \int_{M_{t}}(H-h)^{2} d \mu-\int_{M_{t}}(H-h)^{3} H d \mu .
$$


Now notice that in view of Lemma 3.6(iii)

$$
\frac{d}{d t_{M_{t}}} d \mu=-\int_{M_{t}}(H-h)^{2} d \mu
$$

such that

$$
\int_{0}^{\infty} \int_{M_{t}}(H-h)^{2} d \mu d t \leqq\left|M_{0}\right|
$$

is uniformly bounded. Thus, in view of our higher derivative estimates $\max _{M_{t}}$ $|H-h|$ tends to zero as $t \rightarrow \infty$, in particular there is $t_{0}$ such that the estimate $\max |H(H-h)| \leqq \varepsilon m \sigma^{-3}$ holds for $t>t_{0}$. Therefore we conclude that

$$
\frac{d}{d t} \int_{M_{t}}(H-h)^{2} d \mu \leqq-\frac{(12-2 \varepsilon) m}{\sigma^{3}} \int_{M_{t}}(H-h)^{2} d \mu
$$

which implies exponential decay of this $L^{2}$-integral. Exponential convergence in higher norms now follows from standard interpolation inequalities, completing the proof of Theorem 3.1.

3.14 Remark. (i) Instead of the centered sphere $S_{\sigma}(0)$ we could have used any sphere $S_{\sigma}(\vec{a})$ as initial surface for the flow with this proof, as long as $|\vec{a}|$ is sufficiently small compared to $\sigma$. With some additional work using the estimates in Sect. 5 it is also possible to prove that round initial spheres displaced by a distance just slightly smaller than $\sigma$ drift back to the center under the flow.

(ii) If the mass is negative, any sphere which is 'off center' initially, will drift further away under this flow, compare the expression for the mean curvature in Proposition 2.2.

(iii) Although we have only presented the three-dimensional case here, the existence proof clearly carries over to asymptotically flat manifolds with positive mass in higher dimensions.

\section{The Foliation}

In this section we prove that the constant mean curvature surfaces constructed in the previous section are stable and form a proper foliation of $N \backslash B_{\sigma_{0}}(0)$, provided $\sigma_{0}$ is sufficiently large. We then prove that this foliation is at least locally unique and has a common center of gravity as the radius of the surfaces tends to infinity. Global uniqueness results will be obtained in section 5 .

For $\sigma \geqq \sigma_{0}$ let $M_{\sigma}$ be the constant mean curvature surface constructed in Theorem 3.1 and denote by $H_{\sigma}$ its mean curvature. We say that $M$ is stable if volume preserving variations of $M$ in $N$ do not decrease the area and we say that $M$ is strictly stable if the second variation operator on $M$,

$$
L u=-\Delta u-\left(|A|^{2}+\bar{R} i c(v, v)\right) u
$$


has only strictly positive eigenvalues when restricted to functions $u$ with $\int u d \mu=0$. Then we have the following result on the existence of a stable foliation by constant mean curvature surfaces.

4.1 Theorem. There is $\sigma_{0}$ depending only on $m>0$ and $C_{0}$ such that the constant mean curvature surfaces $M_{\sigma}$ constructed in Theorem 3.1, $\sigma \geqq \sigma_{0}$, form a proper foliation of $N \backslash B_{\sigma_{0}}(0)$. Each $M_{\sigma}$ is strictly stable, the lowest eigenvalue of the stability operator $L$ on volume preserving deformations is of order $6 m \sigma^{-3}$. Moreover, given constants $B_{1}, B_{2}, B_{3}$ one can choose $\sigma \geqq$ $\sigma_{0} \geqq c\left(C_{0}^{2}+B_{1}^{2}+B_{2}+B_{3}\right)$ such that $M_{\sigma}$ is the only surface with constant mean curvature $H_{\sigma}$ contained in $B_{\sigma}\left(B_{1}, B_{2}, B_{3}\right)$.

Proof. Suppose that $B_{1}, B_{2}, B_{3}$ and $\sigma_{0} \geqq c\left(C_{0}^{2}+B_{1}^{2}+B_{2}+B_{3}\right)$ are such that our previous results, in particular Theorem 3.3, Propositions 2.1,2.2 and Lemma 3.13 all apply. Then we have

$$
\left|\left(|A|^{2}+\bar{R} i c(v, v)\right)-\frac{2}{\sigma^{2}}+\frac{10 m}{\sigma^{3}}\right| \leqq c\left(C_{0}+B_{1}+B_{2}+B_{3}\right) \sigma^{-4}
$$

on $M_{\sigma}$, such that in view of Lemma 3.13 the lowest eigenvalue of the operator $L$ restricted to functions $\mathrm{u}$ with $\int u d \mu=0$ is of order $6 m \sigma^{-3}$. This proves the stability of $M_{\sigma}$ and it is well known that then the $M_{\sigma}$ form a foliation. To obtain stronger control of the foliation, we examine the operator $L$ now more closely.

Let $\mu_{0}$ be the only negative eigenvalue of $L$ with eigenfunction $h_{0}$, i.e.

$$
\Delta h_{0}+\left(|A|^{2}+\bar{R} i c(v, v)\right) h_{0}=-\mu_{0} h_{0} .
$$

Multiplying with $h_{0}$ and integrating we see from (4.1) that

$$
\mu_{0} \geqq-\frac{2}{\sigma^{2}}+\frac{10 m}{\sigma^{3}}-c\left(C_{0}+B_{1}+B_{2}+B_{3}\right) \sigma^{-4} .
$$

On the other hand $\mu_{0}$ is characterised as

$$
\mu_{0}=\inf _{\|f\|_{2}=1} \int|\nabla f|^{2}-\left(|A|^{2}+\bar{R} i c(v, v)\right) f^{2} d \mu .
$$

Choosing $f \equiv$ const. as a comparison function we get the reverse inequality

$$
\mu_{0} \leqq-\frac{2}{\sigma^{2}}+\frac{10 m}{\sigma^{3}}+c\left(C_{0}+B_{1}+B_{2}+B_{3}\right) \sigma^{-4} .
$$

To show that $h_{0}$ is close to a constant function, let $\bar{h}_{0}=\oint h_{0} d \mu$ be the mean value of $h_{0}$ on $M_{\sigma}$. After multiplying (4.2) with $\left(h_{0}-\bar{h}_{0}\right)$ and integration by parts we obtain

$$
\begin{aligned}
\int|\nabla h|^{2} d \mu= & \int\left(\mu_{0}+|A|^{2}+\bar{R} i c(v, v)\right)\left(h_{0}-\bar{h}_{0}\right)^{2} d \mu \\
& +\bar{h}_{0} \int\left(|A|^{2}+\bar{R} i c(v, v)\right)\left(h_{0}-\bar{h}_{0}\right) d \mu .
\end{aligned}
$$


Since by Lemma 3.13

$$
\int\left|\nabla h_{0}\right|^{2} d \mu=\int\left|\nabla\left(h_{0}-\bar{h}_{0}\right)\right|^{2} d \mu \geqq{ }_{2}^{3} \sigma^{-2} \int\left(h_{0}-\bar{h}_{0}\right)^{2} d \mu,
$$

we obtain from (4.3)-(4.5)

$$
\int\left(h_{0}-\bar{h}_{0}\right)^{2} d \mu \leqq C_{1}\left|\bar{h}_{0}\right| \sigma^{-2} \int\left|h_{0}-\bar{h}_{0}\right| d \mu,
$$

where $C_{1}$ depends on $C_{0}, B_{1}, B_{2}$ and $B_{3}$. So we conclude that

$$
\int\left(h_{0}-\bar{h}_{0}\right)^{2} d \mu \leqq c C_{1}^{2} \sigma^{-4} \int\left|\bar{h}_{0}\right|^{2} d \mu .
$$

Standard linear estimates then show that also

$$
\sup \left|h_{0}-\bar{h}_{0}\right| \leqq c C_{1} \sigma^{-2}\left|\bar{h}_{0}\right|,
$$

in particular $h_{0}$ doesn't change sign for $\sigma \geqq \sigma_{0}$ large enough. Now let $\mu_{1}$ be the next eigenvalue of $L_{\sigma}$ with corresponding eigenfunction $h_{1}$. To see now that the mean value $\bar{h}_{1}=\oint h_{1} d \mu$ is very small, observe that $h_{1}$ is orthogonal to $h_{0}$ and therefore

$$
0=\int h_{0} h_{1} d \mu=\int h_{1}\left(h_{0}-\bar{h}_{0}\right) d \mu+\bar{h}_{0} \int h_{1} d \mu .
$$

Hence we get from (4.6)

$$
\left|\int h_{1} d \mu\right| \leqq c C_{1} \sigma^{-2} \int\left|h_{1}\right| d \mu .
$$

Now multiply the equation

$$
\Delta h_{1}+\left(|A|^{2}+\bar{R} i c(v, v)\right) h_{1}=-\mu_{1} h_{1}
$$

with $\left(h_{1}-\bar{h}_{1}\right)$ and integrate. Then, since the lowest eigenvalue of $L$ restricted to functions with zero mean value is $6 m \sigma^{-3}$ up to terms of order $\sigma^{-4}$, we derive from (4.1)

$$
\begin{aligned}
(6-\varepsilon) m \sigma^{-3} & \int\left(h_{1}-\bar{h}_{1}\right)^{2} d \mu \\
& \leqq \mu_{1} \int\left(h_{1}-\bar{h}_{1}\right)^{2} d \mu+\bar{h}_{1} \int\left(|A|^{2}+\bar{R} i c(v, v)\right)\left(h_{1}-\bar{h}_{1}\right) d \mu \\
& \leqq \mu_{1} \int\left(h_{1}-\bar{h}_{1}\right)^{2} d \mu+c C_{1} \sigma^{-4}\left|\bar{h}_{1}\right| \int\left|h_{1}-\bar{h}_{1}\right| d \mu \\
& \leqq \mu_{1} \int\left(h_{1}-\bar{h}_{1}\right)^{2} d \mu+c C_{1} \sigma^{-6} \int\left(h_{1}-\bar{h}_{1}\right)^{2} d \mu
\end{aligned}
$$

such that $\mu_{1} \geqq(6-\varepsilon) m \sigma^{-3}$ provided $\sigma \geqq \sigma_{0} \geqq c\left(C_{0}^{2}+B_{1}^{2}+B_{2}+B_{3}\right)$ is large. Thus we have now shown that the operator $L$ is invertible with a bound for the inverse operator $L^{-1}$ given by $\mathrm{cm}^{-1} \sigma^{-3}$.

To complete the proof of Theorem 4.1 it remains only to show that the surface $M_{\sigma}$ constructed in Theorem 3.1 is the only constant mean curvature surface with mean curvature $H_{\sigma}$ in the neighbourhood of $M_{\sigma}$ given by $B_{\sigma}\left(B_{1}, B_{2}, B_{3}\right)$.

Consider the smooth operator $\mathscr{H}: C^{3}\left(S^{2}, R^{3}\right) \rightarrow C^{1}\left(S^{2}\right)$ which assigns to each embedding $F: S^{2} \rightarrow R^{3}$ the mean curvature $\mathscr{H}(F)$ of $F\left(S^{2}\right)$. Given 
a variation vectorfield $V$ on a constant mean curvature surface $M$ it is well known that the derivative of $\mathscr{H}$ at $M$ in direction $V$ is given by

$$
d \mathscr{H}(F) \cdot V=-\Delta\langle V, v\rangle-\left(|A|^{2}+\bar{R} i c(v, v)\right)\langle V, v\rangle=L\langle V, v\rangle,
$$

where $F$ and $L$ denote the imbedding of $M$ and the second variation operator on $M$ respectively. The tangential component of $V$ doesn't yield any contribution since the mean curvature of $M$ is constant.

Suppose now there is another surface $M_{\sigma^{\prime}}$ in $B_{\sigma}\left(B_{1}, B_{2}, B_{3}\right)$ with constant mean curvature $H_{\sigma}$. If $F_{0}, F_{1}: S^{2} \rightarrow R^{3}$ denote the embedding of $M_{\sigma}$ and $M_{\sigma^{\prime}}$ respectively, then we see from Proposition 2.1 that there is a vector $\vec{a}$ such that the second surface can be represented by a normal variation of the form

$$
F_{1}(p)=F_{0}(p)+u(p) v(p), \quad p \in S^{2},
$$

where $u=\langle\vec{a}, v\rangle+G$, and $G$ satisfies the estimate

$$
\sigma|G|+\sigma^{2}|\nabla G|+\sigma^{3}\left|\nabla^{2} G\right|+\sigma^{4}\left|\nabla^{3} G\right| \leqq c\left(C_{0}+B_{1}+B_{2}+B_{3}\right) .
$$

If we then consider the one-parameter family of surfaces $M_{t}$ given by

$$
F_{t}=F_{0}+t u v,
$$

we see that all interpolated surfaces are still in $B_{\sigma}\left(B_{1}, B_{2}, B_{3}\right)$ such that the operator L satisfies the eigenvalue estimates established above on all these surfaces. Since $\mathscr{H}\left(F_{0}\right)=\mathscr{H}\left(F_{1}\right) \equiv H_{\sigma}$ we get from Taylor's theorem that the variation vectorfield $V=F_{1}-F_{0}$ satisfies both

$$
\left\|d \mathscr{H}\left(F_{0}\right) \cdot \vee\right\| \leqq \sup _{t \in[0,1]}\left\|d^{2} \mathscr{H}(F(t))(\vee, \vee)\right\|
$$

and

$$
\left\|d \mathscr{H}\left(F_{0}\right) \cdot \vee\right\| \leqq \sup _{t \in[0,1]}\left\|\left(d \mathscr{H}(F(t))-d \mathscr{H}\left(F_{0}\right)\right) \cdot \vee\right\| .
$$

We have already seen that $L$ ist invertible with $\left\|L^{-1}\right\| \leqq \mathrm{cm}^{-1} \sigma^{3}$. On the other hand it is clear from Proposition 2.1 and (4.7) that the second variation of the mean curvature, which involves cubic terms in the second fundamental form and higher derivatives of the metric, satisfies $\left\|d^{2} \mathscr{H}(\vee, \vee)\right\| \leqq c C_{0} \sigma^{-3}\|\vee\|^{2}$. Hence we see from (4.8) that there is an absolute constant $c_{3}$ such that $\|\vee\| \leqq$ $c_{3} C_{0} m^{-1}$ implies $\vee \equiv 0$. But in view of (4.7) this means that $|\vec{a}| \leqq c_{3} C_{0} m^{-1}$ implies $F_{0} \equiv F_{1}$ provided $\sigma \geqq \sigma_{0} \geqq c\left(C_{0}+B_{1}+B_{2}+B_{3}\right)$ is sufficiently large. We will now use the inequality (4.9) to show that the bound $|\vec{a}| \leqq c_{3} C_{0} m^{-1}$ is true for suitably large $\sigma \geqq \sigma_{0}$. First observe that for all $t \in[0,1]$

$$
\begin{aligned}
d \mathscr{H}(F(t)) \cdot \vee & =d \mathscr{H}(F(t)) \cdot \vee^{\perp}+d \mathscr{H}(F(t)) \cdot \vee^{\top} \\
& =L_{t}\langle\vee, v\rangle+\vee^{\top}\left(H^{t}\right) .
\end{aligned}
$$

Here $\perp$ and $\top$ denote the tangential and vertical component of a vector with respect to $F(t)\left(S^{2}\right), L_{t}$ is the second variation operator on $F(t)\left(S^{2}\right)$ and $\vee^{\top}\left(H^{t}\right)$ 
is the derivative of the mean curvature on $F(t)\left(S^{2}\right)$ in direction $\vee^{\top}$. For a surface in $B_{\sigma}\left(B_{1}, B_{2}, B_{3}\right)$ the second term on the RHS can be estimated by

$$
\left\|\vee^{\top}\left(H^{t}\right)\right\| \leqq c B_{3} \sigma^{-4} \text {. }
$$

Since

$$
L_{t}\langle\vee, v\rangle=-\Delta\langle\vee, v\rangle-\left(|A|^{2}+\bar{R} i c(v, v)\right)\langle\vee, v\rangle
$$

and since $\vee=\vec{a}+G$, we get from (4.1) and (4.7) that

$$
\|(d \mathscr{H}(F(t))-d \mathscr{H}(F(0))) \vee\| \leqq c \sigma^{-4}\left(C_{0}+B_{1}+B_{2}+B_{3}\right) .
$$

Since on the other hand $\langle\vec{a}, v\rangle$ is an approximate eigenfunction of $L$ with eigenvalue close to $6 m \sigma^{-3}$ we have $\left\|d \mathscr{H}\left(F_{0}\right) \cdot \vee\right\| \geqq m \sigma^{-3}|\vec{a}|$. Applying now inequality (4.9) we see that for $\sigma \geqq \sigma_{0} \geqq c\left(B_{1}+B_{2}+B_{3}+C_{0}\right) m^{-1}$ we must have $|\vec{a}| \leqq c_{3} C_{0} m^{-1}$ and thus $F_{0} \equiv F_{1}$. This completes the proof of Theorem 4.1.

We will now show that the surfaces $M_{\sigma}$ have a joint 'center of gravity' as $\sigma$ tends to infinity.

4.2 Theorem. There is a vector $\vec{a} \in \mathbf{R}^{3}$ depending only on $N$ such that the center of gravity of $M_{\sigma}$ with respect to $\mathbf{R}^{3}$ converges to $\vec{a}$ as $\sigma \rightarrow \infty$.

Proof. It will be convenient in this proof to label the surfaces $M_{\sigma}$ by their mean curvature: We write $\tau(\sigma)=H_{\sigma}$ and $M(\tau)$ for the surface $M_{\sigma}$ with $H_{\sigma}=\tau$. $M(\tau)$ is uniquely determined by Theorem 4.1 and we write

$$
\vec{g}_{\tau}=\int_{M(\tau)} F(\tau) d \mu_{e} / \int_{M(\tau)} d \mu_{e}
$$

for the center of gravity of $M(\tau)$ with respect to the Euclidean metric. Here $F(\tau)$ is the embedding of $M(\tau)$ and we want to show that $-(d / d \tau) \vec{g}_{\tau}$ is small. So we have to study the solution $u$ of the equation

$$
-L u=\Delta u+\left(|A|^{2}+\bar{R} i c(v, v)\right) u=1
$$

describing the lapse function of the foliation on $M_{\tau}$. Let $h_{0}$ be the first eigenfunction of $L$ as before and normalize $h_{0}$ such that $\oint h_{0} d \mu=\bar{h}_{0}=\oint u d \mu=\bar{u}$. Then

$$
\Delta\left(u-h_{0}\right)+\left(|A|^{2}+\bar{R} i c(v, v)\right)\left(u-h_{0}\right)=1-\mu_{0} h_{0} .
$$

Now let $\sigma$ be such that $\tau(\sigma)=\tau$, and multiply both sides with $\left(u-h_{0}\right)$. Since the lowest eigenvalue of $\mathrm{L}$ on functions of zero mean value is larger than $\mathrm{cm} \sigma^{-3}$, we derive after integration by parts

$$
3 m \sigma_{1}^{-3} \int_{M(\tau)}\left(u-h_{0}\right)^{2} d \mu \leqq-\mu_{0} \int_{M(\tau)}\left(h_{0}-\bar{h}_{0}\right)\left(u-h_{0}\right) d \mu .
$$

Since $\sigma_{0}, B_{1}, B_{2}, B_{3}$ have been chosen uniformly in $\sigma$ at this stage, we see from (4.6) that there is a constant $C_{4}$ depending only on $m^{-1}$ and $C_{0}$ such that

$$
\left|h_{0}-\bar{h}_{0}\right| \leqq C_{4} \sigma^{-2}\left|\bar{h}_{0}\right| \text {. }
$$


Thus, using also the fact that $\left|h_{0}\right| \leqq c \sigma^{2}$ we obtain

$$
\int_{M(\tau)}\left(u-h_{0}\right)^{2} d \mu \leqq C_{4} \sigma^{2}
$$

and standard linear estimates then show that also sup $\left|h_{0}-u\right| \leq C_{4}$. Hence we finally have

$$
\sup |u-\bar{u}| \leqq \sup \left(\left|u-h_{0}\right|+\left|h_{0}-\bar{h}_{0}\right|\right) \leq C_{4}
$$

for some constant $C_{4}$ depending only on $\mathrm{m}^{-1}$ and $C_{0}$. In view of Proposition 2.1 the normal of $M_{\tau}$ with respect to the metric $\mathrm{g}$ differs from the Euclidean unit normal by a term of order $\sigma^{-2}$, hence we easily see that

$$
\left|\frac{d}{d \tau} \vec{g}_{\tau}\right| \leqq C
$$

Furthermore, since $|d \tau / d \sigma| \leqq c \sigma^{-2}$, we conclude that

$$
\left|\frac{d}{d \sigma} \vec{g}_{\tau(\sigma)}\right| \leqq c \sigma^{-2}
$$

giving the desired conclusion.

4.3 Remark. The estimates established here show that the foliation can be used to define a natural polar coordinate system at infinity: A radial coordinate $\tilde{r}(\sigma)$ is given by $\left|M_{\sigma}\right|=4 \pi \tilde{r}_{\sigma}^{2}$, and angular coordinates can be defined since the estimates ensure convergence of the unit normal on $M_{\sigma}$ for $\sigma \rightarrow \infty$ to points on the Euclidean sphere at infinity.

\section{Global Uniqueness}

In this section we will show that the foliation $\left\{M_{\sigma}\right\}$ constructed in Theorem 3.1 and Theorem 4.1 is unique. In fact, we show that the individual surfaces $M_{\sigma}$ are the only stable constant mean curvature surfaces outside a small interior region of $N$.

5.1 Theorem. Let $m>0$ and $1 / 2<q \leqq 1$ be given. Then there is $H_{0}>0$ depending only on $m^{-1}, q$ and $C_{0}$ such that for all $0<H<H_{0}$ the leaf $M_{H}$ of the foliation constructed in Theorem 4.1 is the only surface of constant mean curvature $H$, which is stable and contained in $N \backslash B_{H^{-q}}(0)$.

Note that no curvature bound for the hypersurface is assumed in this result, and the interior radius $H^{-q}$ is much smaller than the diameter of $M_{H}$ if $q<1$. The exponent $q>1 / 2$ seems to be optimal from a technical point of view, but it is an open question whether stable constant mean curvature surfaces are actually completely unique outside a fixed compact set. The stability assumption is only used to derive curvature estimates and can be discarded if only surfaces are considered where the principal curvatures are comparable to ${ }_{2}^{1} \mathrm{H}$. 
Using suitable testfunctions for the stability operator we will first show that any stable constant mean curvature surface satisfies an estimate on an $L^{2}$ - curvature integral. It is then shown that it is "round" in the sense of Sect. 2 and has to lie in some $B_{\sigma}\left(B_{1}, B_{2}, B_{3}\right)$ where the uniqueness result in Theorem 4.1 can be applied. Let $S$ in the following be a stable hypersurface of constant mean curvature $H$ which is contained in $N \backslash B_{\sigma_{0}}(0)$. We begin with a technical lemma.

5.2 Lemma. Let $X=x_{i}\left(\partial / \partial x_{i}\right)$ be the Euclidean coordinate vectorfield and let as before $r=\left(\sum x_{1}^{2}\right)^{1 / 2}$. Then for $\sigma_{0} \geqq c C_{0}$ we have the estimate

$$
\int_{S}\langle X, v\rangle^{2} r^{-4} d \mu \leqq H^{2}|S| .
$$

Moreover, for each $\alpha \geqq \alpha_{0}>2$ and $\sigma_{0} \geqq c C_{0}\left(\alpha_{0}-2\right)^{-1}$ sufficiently large we have the estimate

$$
\int_{S} r^{-\alpha} d \mu \leqq c\left(\alpha_{0}\right) \sigma_{0}^{(2-\alpha)} H^{2}|S|
$$

Proof. Since $S$ has constant mean curvature $H$, the divergence formula takes the form

$$
\int_{S} \operatorname{div}_{S} Y d \mu=H \int_{S}\langle Y, v\rangle d \mu .
$$

Now choose the vectorfield $Y=X r^{-\alpha}, \alpha \geqq 2$ and observe that

$$
\begin{gathered}
\operatorname{div}_{S} Y=r^{-\alpha} \operatorname{div}_{S} X-\alpha r^{-\alpha-2}\left|X^{\tau}\right|^{2}, \\
\left|\operatorname{div}_{S} X-2\right| \leqq c C_{0} r^{-1} .
\end{gathered}
$$

We then obtain

$$
\left|(2-\alpha) \int_{S} r^{-\alpha} d \mu+\alpha \int_{S}\langle X, v\rangle^{2} r^{-\alpha-2} d \mu-H \int_{S}\langle X, v\rangle r^{-\alpha} d \mu\right| \leqq c C_{0} \int_{S} r^{-\alpha-1} d \mu .
$$

Choosing first $\alpha=2$ this yields

$$
\int_{S}\langle x, v\rangle^{2} r^{-4} d \mu \leqq{ }_{4}^{1} H^{2}|S|+c C_{0} \int_{S} r^{-3} d \mu .
$$

Using (5.1) now with $\alpha=3$ we derive

$$
\int_{S} r^{-3} d \mu \leqq 4 \sigma_{0}^{-1} \int_{S}\langle x, v\rangle^{2} r^{-4} d \mu+\sigma_{0}^{-1} H^{2}|S|+c C_{0} \sigma_{0}^{-1} \int_{S} r^{-3} d \mu .
$$

Thus, choosing $\sigma \geqq c C_{0}$ sufficiently large, and combining this inequality with (5.2) we obtain (i). Now let $\alpha \geqq \alpha_{0}>2$ be arbitrary in (5.1) to conclude

$$
\int_{S} r^{-\alpha} d \mu \leq c\left(\alpha_{0}-2\right)^{-1} \sigma_{0}^{(2-\alpha)}\left(\int_{S}\langle x, v\rangle^{2} r^{-4} d \mu+H^{2}|S|+c C_{0} \int_{S} r^{-3} d \mu\right) .
$$

The desired estimate then follows from (i). 
In a first step we now use the stability assumption to prove an integral estimate for the difference of the principal curvatures on $S$.

5.3 Proposition. Suppose that $S$ is stable. Then we have for $\sigma_{0} \geqq c C_{0}$ the estimate

$$
\int_{S}\left(\lambda_{1}-\lambda_{2}\right)^{2} d \mu \leqq c C_{0} \sigma_{0}^{-1} H^{2}|S| \leqq \tilde{C} \sigma_{0}^{-1} .
$$

Proof. Since $S$ is stable, we have

$$
\int_{S}|\nabla f|^{2} d \mu-\int_{S}\left(|A|^{2}+\bar{R} i c(v, v)\right) f^{2} d \mu \geq 0
$$

for any function $f$ with $\int_{S} f d \mu=0$. We follow an idea in $[C Y]$ and choose $\psi$ to be a conformal map of degree 1 which maps $S$ onto the standard sphere $S^{2}$ in Euclidean space $\mathbf{R}^{3}$. Using the conformal group of $S^{2}$ it can be arranged that each component $\psi_{i}$ of $\psi$ satisfies $\int \psi_{i} d \mu=0$, see [CY] and $[L Y]$. The Dirichlet integral in (5.3) is equal to $8 \pi / 3$ for each $f=\psi_{i}$. Since $\sum \psi_{i}^{2} \equiv 1$ we conclude that

$$
8 \pi \geqq \int_{S}|A|^{2}+\bar{R} i c(v, v) d \mu .
$$

Again following $[\mathrm{CY}]$ we can now use the Gauß equation to obtain

$$
|A|^{2}+\bar{R} i c(v, v)={ }_{2}^{1}\left(|A|^{2}-{ }_{2}^{1} H^{2}\right)+{ }_{4}^{3} H^{2}+{ }_{2}^{1} \bar{R}-K,
$$

where $\mathrm{K}$ is the Gauß curvature of $\mathrm{S}$ and $\bar{R}$ is the scalar curvature of $N$. Combining (5.4) and (5.5) we derive in view of $|A|^{2}-{ }_{2}^{1} H^{2}=\frac{1}{2}\left(\lambda_{1}-\lambda_{2}\right)^{2}$ that

$$
\int_{S}\left(\lambda_{1}-\lambda_{2}\right)^{2} d \mu+3 \int_{S} H^{2} d \mu \leqq 48 \pi-2 \int_{S} \bar{R} d \mu .
$$

Since $|\bar{R}| \leqq c C_{0} r^{-3}$, Lemma 5.2.(ii) then implies that

$$
H^{2}|S| \leqq C,
$$

with a uniform constant $\mathrm{C}$ provided $\sigma_{0} \geq c C_{0}$ is large enough. We may now combine (5.4) and (5.5) slightly differently to compute

$$
\begin{aligned}
\int_{S}\left(\lambda_{1}-\lambda_{2}\right)^{2} d \mu & =\int_{S} 2|A|^{2}-H^{2} d \mu \\
& =\int_{S}|A|^{2}+\bar{R} i c(v, v) d \mu+\int_{S} \bar{R}-3 \bar{R} i c(v, v)-2 K d \mu \\
& \leqq 8 \pi+\int_{S} \bar{R}-3 \bar{R} i c(v, v) d \mu-8 \pi \\
& =\int_{S} \bar{R}-3 \bar{R} i c(v, v) d \mu \\
& \leqq c C_{0} \int_{S} r^{-3} d \mu .
\end{aligned}
$$


The desired estimate then follows from Lemma 5.2 (ii) and (5.7). To proceed further, we use the second variation inequality (5.3) as in [SSY] with the testfunction

$$
f=u-\oint_{S} u d \mu \equiv u-\bar{u}, \quad u=|\stackrel{\circ}{A}| .
$$

With this choice of $f$ we derive

$$
\begin{aligned}
\int_{S}\left(|A|^{2}+\right. & \bar{R} i c(v, v))|\stackrel{\circ}{A}|^{2} d \mu \\
& \leqq \int_{S}\left(2 u \bar{u}-\bar{u}^{2}\right)\left(|A|^{2}+\bar{R} i c(v, v)\right) d \mu+\int_{S}|\nabla| \stackrel{\circ}{A} \|^{2} d \mu .
\end{aligned}
$$

Since the mean curvature is constant we may proceed as in $[S S Y ; 1.34]$ to derive the following inequality, where we also use the fact that $|\bar{R} i c| \leqq c C_{0} r^{-3}$ and $|\bar{\nabla} \bar{R} i c| \leqq c C_{0} r^{-4}$.

$$
\begin{aligned}
|\stackrel{\circ}{A}| \Delta|\stackrel{\circ}{A}| \geqq & \left(H^{2}-|A|^{2}\right)|\stackrel{\circ}{A}|^{2}+\left.\frac{1}{1+\varepsilon}|\nabla| \stackrel{\circ}{A}\right|^{2}-\frac{c}{1+\varepsilon} C_{0} r^{-6} . \\
& -c C_{0}|\stackrel{\circ}{A}| r^{-4}-c C_{0} r^{-3}|\stackrel{\circ}{A}|^{2}-c C_{0} H|\stackrel{\circ}{A}| r^{-3}
\end{aligned} .
$$

Here $\varepsilon>0$ is arbitrary and integration yields

$$
\begin{aligned}
\left.\left(1+\frac{1}{1+\varepsilon}\right) \int_{S}|\nabla| \stackrel{\circ}{A}\right|^{2} d \mu & \leqq \int_{S}\left(|A|^{2}-H^{2}\right)|\stackrel{\circ}{A}|^{2} d \mu \\
& +c C_{0} \int_{S}\left(|\stackrel{\circ}{A}|^{2}+H|\stackrel{\circ}{A}|+|\stackrel{\circ}{A}|^{-1}+\varepsilon^{-1} r^{-3}\right) r^{-3} d \mu .
\end{aligned}
$$

Multiply (5.8) with $2 /(1+\varepsilon)$ and add the last inequality to derive

$$
\begin{aligned}
& \left(\begin{array}{c}
1-\varepsilon \\
1+\varepsilon
\end{array}\right) \int_{S}|A|^{2}|\stackrel{\circ}{A}|^{2} d \mu+\left.\frac{\varepsilon}{1+\varepsilon} \int_{S}|\nabla| \stackrel{\circ}{A}\right|^{2} d \mu \\
& \leqq \\
& \int_{S}\left(2 u \bar{u}-\bar{u}^{2}\right)\left(|A|^{2}+\bar{R} i c(v, v)\right) d \mu-\int_{S} H^{2}|\stackrel{\circ}{A}|^{2} d \mu \\
& \quad+c C_{0} \int_{S}\left(|\stackrel{\circ}{A}|^{2}+H|\stackrel{\circ}{A}|+|\stackrel{\circ}{A}| r^{-1}+\varepsilon^{-1} r^{-3}\right) r^{-3} d \mu .
\end{aligned}
$$

Now notice that $|A|^{2}=|\stackrel{\circ}{A}|^{2}+{ }_{2}^{1} H^{2}$ and $2 u \bar{u} \leqq \bar{u}^{2}+|\stackrel{\circ}{A}|^{2}$ such that

$$
\begin{aligned}
\left(\begin{array}{c}
1-\varepsilon \\
1+\varepsilon
\end{array}\right) \int_{S}|\stackrel{\circ}{A}|^{4} d \mu & +\frac{(1-\varepsilon)}{(1+\varepsilon)} H^{2} \int|\stackrel{\circ}{A}|^{2} d \mu+\left.\frac{\varepsilon}{1+\varepsilon} \int_{S}|\nabla| \stackrel{\circ}{A}\right|^{2} d \mu \\
\leqq & \int_{S}\left(2 u \bar{u}-\bar{u}^{-2}\left(|\stackrel{\circ}{A}|^{2}+\bar{R} i c(v, v)\right) d \mu\right. \\
& +c C_{0} \int_{S}\left(|\stackrel{\circ}{A}|^{2}+H|\stackrel{\circ}{A}|+|\stackrel{\circ}{a}| r^{-1}+\varepsilon^{-1} r^{-3}\right) r^{-3} d \mu .
\end{aligned}
$$


Since $|\bar{R} i c(v, v)| \leqq c C_{0} r^{-3}$, the RHS can be estimated by

$$
2 \bar{u} \int_{S}|\stackrel{\circ}{A}|^{3} d \mu+c C_{0} \int_{S}\left(|\stackrel{\circ}{A}|^{2}+H|\stackrel{\circ}{A}|+|\stackrel{\circ}{A}| r^{-1}+\varepsilon^{-1} r^{-3}\right) r^{-3} d \mu .
$$

By Hölder's inequality and Proposition 5.3

$$
\bar{u}^{2}=|S|^{-2}\left(\int_{S}|\stackrel{\circ}{A}| d \mu\right)^{2} \leqq|S|^{-1} \int|\stackrel{\circ}{A}|^{2} d \mu \leqq C C_{0} \sigma_{0}^{-1} H^{2}
$$

such that

$$
2 \bar{u} \int_{S}|\stackrel{\circ}{A}|^{3} d \mu \leqq \frac{1}{2} \int_{S}|\stackrel{\circ}{A}|^{4} d \mu+c C_{0} \sigma_{0}^{-1} H^{2} \int_{S}|\stackrel{\circ}{A}| d \mu .
$$

Choosing then $\sigma \geqq c C_{0}$ sufficiently large and $0<\varepsilon<\frac{1}{2}$ suffienctly small we conclude

$$
\begin{array}{r}
\int|\stackrel{\circ}{A}|^{4} d \mu+H^{2} \int_{S}|\stackrel{\circ}{A}|^{2} d \mu+\left.\int_{S}|\nabla| \stackrel{\circ}{A}\right|^{2} d \mu \leqq \\
c C_{0} \int_{S}\left(|\stackrel{\circ}{A}|^{2}+H|\stackrel{\circ}{A}|+|\stackrel{\circ}{A}| r^{-1}+r^{-3}\right) r^{-3} d \mu .
\end{array}
$$

Using now again Hölder's inequality, Lemma 5.2 and Proposition 5.3 we obtain the estimate

$$
\int_{S}|\stackrel{\circ}{A}|^{4} d \mu+H^{2} \int_{S}|\stackrel{\circ}{A}|^{2} d \mu+\left.\int_{S}|\nabla| \stackrel{\circ}{A}\right|^{2} d \mu \leqq c C_{0} \sigma_{0}^{-4} .
$$

At this stage we need a Sobolev inequality on S.

5.4 Proposition. There is an absolute constant $c$ such that provided $\sigma_{0} \geqq c C_{0}$ sufficiently large we have for all Lipschitz functions $g$ on $S$ the Sobolev inequality

$$
\left(\int_{S}|g|^{2} d \mu\right)^{\frac{1}{2}} \leqq c \quad \int_{S}|\nabla g|+H|g| d \mu .
$$

Proof. It is wellknown that the above inequality holds for hypersurfaces S immersed in Euclidean space if $H$ is replaced by the mean curvature $H^{e}$ of $\mathrm{S}$ with respect to the Euclidean metric. From Lemma 1.4 and (5.6) it is clear that the difference between $H^{e}$ and $H$ can be bounded by $c C_{0} r^{-2}$. Since the metric $g_{\alpha \beta}$ and the Euclidean metric are uniformly equivalent on $N$ this shows that

$$
\left(\int_{S}|g|^{2} d \mu\right)^{\frac{1}{2}} \leqq c \int_{S}|\nabla g|+H|g|+C_{0} r^{-2}|g| d \mu
$$


holds with some absolute constant c. Using now Hölder's inequality and Lemma 5.2(ii) we estimate

$$
\begin{aligned}
\int_{S} C_{0} r^{-2}|g| d \mu & \leqq\left(\int_{S}|g|^{2} d \mu\right)^{\frac{1}{2}} \cdot\left(\int_{S} r^{-4} d \mu\right)^{\frac{1}{2}} \\
& \leqq c C_{0} \sigma_{0}^{-1} H|S|^{\frac{1}{2}} \cdot\left(\int_{S}|g|^{2} d \mu\right)^{\frac{1}{2}} .
\end{aligned}
$$

In view of (5.7) this term can be absorbed on the LHS for $\sigma_{0} \geqq c C_{0}$ sufficiently large, completing the proof of the Sobolev inequality.

5.5 Corollary. There is an absolute constant $c$ such that provided $\sigma_{0} \geqq c C_{0}$ we have the estimate

$$
c^{-1} H^{-2} \leqq|S| \leqq c H^{-2}
$$

Proof. The second inequality was shown in Proposition 5.3 and the first inequality follows from Proposition 5.4 if we set $g \equiv 1$. Notice also that after replacing $\mathrm{g}$ by $g^{p}, p \geqq 1$ in Proposition 5.4 we obtain from Hölder's inequality that

$$
\left(\int_{S}|g|^{2 p} d \mu\right)^{1 / p} \leqq c p^{2}|\operatorname{supp} g|^{1 / p} \cdot \int_{S}|\nabla g|^{2}+H^{2}|g|^{2} d \mu
$$

for all $p \geqq 1$.

We are now ready to derive a pointwise estimate for $u=|\stackrel{\circ}{A}|$. As a first step we prove:

5.6 Lemma. For all $\delta>0$ there is a constant $C\left(\delta, m, C_{0}\right)$ such that

$$
|\stackrel{\circ}{A}| \leqq C\left(\delta, m, C_{0}\right) \sigma_{0}^{-2-\delta} H^{-2 \delta} .
$$

In particular there is $\varepsilon>0$ depending on $q>\frac{1}{2}$ such that

$$
|\stackrel{\circ}{A}| \leqq C\left(q, m, C_{0}\right) H^{1+\varepsilon} \text {. }
$$

Proof. The second inequality follows from the first with $\sigma_{0} \geqq H^{-q}$. Let $u_{k}=$ $\max (u-k, 0)$ and $A(k)=\{x \in S \mid u(x)>k\}$. We multiply inequality (5.9) by $u_{k}^{p}, p \geqq 1$ and integrate by parts. This yields

$$
\begin{aligned}
& \left.\left(\begin{array}{l}
2+\varepsilon \\
1+\varepsilon
\end{array}\right) \int_{A(k)}|\nabla| \stackrel{\circ}{A}\right|^{2} u_{k}^{p} d \mu+\left.p \int_{A(k)} u_{k}^{p-1} u|\nabla| \stackrel{\circ}{A}\right|^{2} d \mu+H^{2} \int_{A(k)} u^{2} u_{k}^{p} d \mu \\
& \leqq \int_{A(k)}|\stackrel{\circ}{A}|^{4} u_{k}^{p} d \mu+c C_{0} \int_{A(k)}\left(u^{2}+u r^{-1}+u H+\varepsilon^{-1} r^{-3}\right) r^{-3} u_{k}^{p} d \mu .
\end{aligned}
$$


Using then $|\stackrel{\circ}{A}|=u, r \geqq \sigma_{0}$, and the Schwarz inequality, we derive

$$
\begin{aligned}
\int_{A(k)}|\nabla u|^{2} u_{k}^{p} d \mu & +p \int_{A(k)} u u_{k}^{p-1}|\nabla u|^{2} d \mu+H^{2} \int_{A(k)} u^{2} u_{k}^{p} d \mu \\
& \leqq \int_{A(k)} u^{4} u_{k}^{p} d \mu+C \int_{A(k)} u_{k}^{p}\left(\sigma_{0}^{-6}+u \sigma_{0}^{-4}\right) d \mu \\
& \leqq \int_{A(k)} u^{4} u_{k}^{p} d \mu+C \sigma_{0}^{-5} \int_{A(k)} u_{k}^{p} d \mu .
\end{aligned}
$$

Now we set $f=u_{k}^{p / 2+1}$, such that the above inequality becomes

$$
\frac{1}{p_{A(k)}}|\nabla f|^{2} d \mu+H^{2} \int_{A(k)} u^{2} u_{k}^{p} d \mu \leqq c \int_{A(k)} u^{4} u_{k}^{p} d \mu+C \sigma_{0}^{-4} u_{k}^{p} d \mu .
$$

The Sobolev inequality established above shows that for $1<\eta<\infty$

$$
\begin{aligned}
\left(\int_{A(k)}|f|^{2 \eta} d \mu\right)^{1 / \eta} & \leqq c \eta^{2}|A(k)|^{1 / \eta} \int_{A(k)}|\nabla f|^{2}+H^{2} f^{2} d \mu \\
& \leqq c(p, q)|A(k)|^{1 / \eta} \int_{A(k)} u^{4} u_{k}^{p}+C \sigma_{0}^{-5} u_{k}^{p} d \mu,
\end{aligned}
$$

such that by the Hölder inequality

$$
\int_{A(k)} f^{2} d \mu \leqq c(p, q)|A(k)| \int_{A(k)} u^{4} u_{k}^{p}+C \sigma_{0}^{-5} u_{k}^{p} d \mu .
$$

To estimate the two terms on the RHS of this inequality, observe that for any $\rho \geqq 1$ we have from the Sobolev inequality and estimate (5.11) that

$$
\begin{aligned}
\left(\int_{A(k)} u_{k}^{2 \rho} d \mu\right)^{1 / \rho} & \leqq c \rho^{2}|A(k)|^{1 / \rho}\left(\int_{A(k)}|\nabla u|^{2}+H^{2}|u|^{2} d \mu\right) \\
& \leqq c \rho^{2}|A(k)|^{1 / \rho} C \sigma_{0}^{-4} .
\end{aligned}
$$

Applying this to the first term on the RHS above we get

$$
\int_{A(k)} u^{4} u_{k}^{p} d \mu \leqq \int_{A(k)} u^{p+4} d \mu \leqq C p^{p+4} \sigma_{0}^{-2 p-8}|A(k)|
$$

and from the second term

$$
\int_{A(k)} u_{k}^{p} d \mu \leqq C p^{p} \sigma_{0}^{-2 p}|A(k)| .
$$

We now fix $\eta=2$ to obtain

$$
\int_{A(k)} f^{2} d \mu \leqq C(p) \sigma_{0}^{-2 p-5}|A(k)|^{2}
$$

and since $f^{2}=u_{k}^{p+2}$ we get for $h>k \geqq 0$ the iteration inequality

$$
|h-k|^{p+2}|A(h)| \leqq C|A(k)|^{2} \sigma_{0}^{-2 p-5} .
$$


By a result due to Stampacchia ([St], Lemma 4.1) this implies that $|A(d)|=0$ for

$$
d^{p+2} \leqq C \sigma_{0}^{-2 p-5}|A(0)|
$$

and hence in view of the area estimate for $S$

$$
u \leqq d \leqq C\left(p, m, C_{0}\right) \sigma_{0}^{(-2 p-5) /(p+2)} H^{-2 /(p+2)} .
$$

This yields the desired estimate.

Notice that the inequality just established implies convexity of $S$ and strong pinching of the second fundamental form. We can now bound the derivative of the second fundamental form similarly as in Proposition 3.12.

5.7 Lemma. There is a constant $c$ depending on $q$ such that

$$
|\nabla \AA| \leqq C H^{(3+\varepsilon) / 2} .
$$

Proof. Using the decay properties of the metric and its derivatives we compute as in Corollary 3.11

$$
\Delta|\nabla \AA|^{2} \geqq-c|\nabla \AA|^{2} \sigma_{0}^{-2}-c C_{0}|\nabla \AA| \sigma_{0}^{-5}
$$

Note that the decay of the RHS is slightly worse than in Corollary 3.11 since we do not know yet that $S$ is "centered" and the normal does not necessarily point in an eigendirection of the ambient Ricci curvature. From here we derive as in Proposition 3.12

$$
\triangle\left(|\nabla \AA|^{2}+C_{1}|\AA|^{2} \sigma_{0}^{-2}\right) \geqq c_{1}|\nabla \AA|^{2} \sigma_{0}^{-2}-c C_{0}|\nabla \AA| \sigma_{0}^{-5}-c C_{0}^{2}|\AA| \sigma_{0}^{-6} .
$$

From $|\AA| \leqq C H^{1+\varepsilon}$ and $\sigma_{0} \geqq H^{-q}, q>\frac{1}{2}$ together with the maximum principle we get the desired estimate.

Proof of Theorem 5.1. In view of Lemmas 5.6 and 5.7 the surface $S$ is close to a round Euclidean sphere: Since $\sigma_{0} \geqq H^{-q}$ with $q>\frac{1}{2}$ it follows that the mean curvature $H^{e}$ of $S$ in $\mathbf{R}^{3}$ differs from $H$ by no more than $C H^{1+\varepsilon}$, and proceeding as in Proposition 2.1 we see that there is a vector $\vec{a}$ in $\mathbf{R}^{3}$ and $r_{0}>0$ such that $S$ satisfies

$$
\begin{aligned}
\left|\lambda_{i}{ }^{e}-r_{0}^{-1}\right| & \leqq C H^{1+\varepsilon}, \quad i=1,2, \\
\left|(y-\vec{a})-r_{0} v_{e}\right| & \leqq C H^{-1+\varepsilon}, \\
\left|v_{e}-r_{0}{ }^{-1}(y-\vec{a})\right| & \leqq C H^{\varepsilon} .
\end{aligned}
$$

In the next step we have to show that the displacement $\vec{a}$ is small. For this purpose we think of a variation in direction of $-\vec{a}$ which preserves the enclosed volume and decreases area if $S$ is off center. We begin with the identity

$$
0=H \int_{S}\left\langle\vec{b}, v_{e}\right\rangle_{e} d \mu_{e}=\int_{S} H\left\langle\vec{b}, v_{e}\right\rangle_{e} d \mu_{e}
$$


where $\vec{b}=-\vec{a} /|\vec{a}|$ points in direction of the displacement of the surface. In view of Lemmata 1.2 and 1.4 the mean curvature $H$ can be expressed in terms of $\psi=\left(1+\frac{m}{2 r}\right)^{2}$ and the Euclidean mean curvature $H^{e}$ up to terms of order $r^{-3}$ :

$$
\left|H-\psi^{-1} H^{e}-2 \psi^{-2} \partial_{v_{e}} \psi\right| \leqq C r^{-3} .
$$

So we derive from $(5.13)$ that

$$
\left|\int_{S}\left(H^{e} \psi^{-1}+2 \psi^{-2} \partial_{v_{e}} \psi\right)\left\langle\vec{b}, v_{e}\right\rangle_{e} d \mu_{e}\right| \leqq C \int_{S} r^{-3} d \mu_{e} \leqq C \sigma_{0}^{-1}
$$

where we also used Lemma 5.1 for the last estimate. Using the first variation formula with respect to the Euclidean metric we compute

$$
\int_{S} H^{e} \psi^{-1}\left\langle\vec{b}, v_{e}\right\rangle_{e} d \mu_{e}=\int_{S} d i v_{S}\left(\psi^{-1} \vec{b}\right) d \mu_{e}=-\int_{S} \psi^{-2}\left\langle\vec{b}, \nabla^{e} \psi\right\rangle d \mu_{e} .
$$

Using now $\left\langle\nabla^{e} \psi, \vec{b}\right\rangle=\langle D \psi, \vec{b}\rangle-\left\langle\vec{b}, v_{e}\right\rangle \partial_{v_{e}} \psi$ together with the fact that $|D \psi| \leqq$ $\mathrm{Cr}^{-2}$ we conclude that

$$
\left|\int_{S} 3\left\langle\vec{b}, v_{e}\right\rangle \partial_{v_{e}} \psi d \mu_{e}-\int_{S}\langle\vec{b}, D \psi\rangle d \mu_{e}\right| \leqq C \sigma_{0}^{-1} .
$$

At this stage we utilize that $S$ is close to a Euclidean sphere as described in (5.12) together with $|D \psi| \leqq C r^{-2}$ : We may compute the integrals in (5.16) on the round Euclidean sphere $S_{e}:=S_{r_{0}}^{2}(\vec{a})$, introducing an error no larger than $C H^{\varepsilon}$. Hence, writing $\left\langle\vec{b}, v_{e}\right\rangle=-\cos \varphi, 0 \leqq \varphi \leqq \pi$ and using

$$
\frac{\partial}{\partial x^{i}} \psi=-m\left(1+\frac{m}{2 r}\right) x^{i} r^{-3}
$$

as well as $\left|\vec{x}-\vec{a}-r_{0} v_{e}\right| \leqq C H^{-1+\varepsilon}$ we conclude that up to terms of order $r^{-2} H^{\varepsilon}$

and

$$
\langle\vec{b}, D \psi\rangle=\frac{m}{r^{3}}|\vec{a}|+\frac{m}{r^{3}} r_{0} \cos \varphi
$$

$$
\left\langle\vec{b}, v_{e}\right\rangle \partial_{v_{e}} \psi=\frac{m}{r^{3}}|\vec{a}| \cos ^{2} \varphi+\frac{m}{r^{3}} r_{0} \cos \varphi .
$$

So we derive from (5.16) that

$$
\left|m \int_{S_{e}} \frac{3|\vec{a}|}{r^{3}} \cos ^{2} \varphi+\frac{2 r_{0}}{r^{3}} \cos \varphi-\frac{|\vec{a}|}{r^{3}} d \mu_{e}\right| \leqq C H^{\varepsilon} .
$$

It remains to evaluate the integrals. Using polar coordinates on $S_{e}$ we derive

$$
\int_{S_{e}} \frac{\cos ^{2} \varphi}{r^{3}} d \mu_{e}=2 \pi r_{0}^{2} \int_{0}^{\pi} \sin \varphi \cos ^{2} \varphi r^{-3} d \varphi,
$$

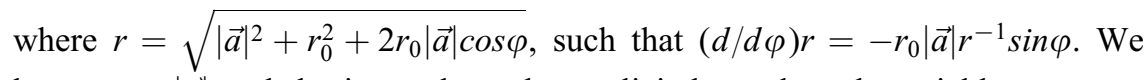
have $r_{0}>|\vec{a}|$ and the integral can be explicitely evaluated to yield

$$
\int_{S_{e}} \frac{\cos ^{2} \varphi}{r^{3}} d \mu_{e}=-\frac{8}{3} \pi \frac{1}{r_{0}}+4 \pi \frac{r_{0}}{r_{0}^{2}-|\vec{a}|^{2}} .
$$


Similarly we compute

$$
\int_{S_{e}} \frac{\cos \varphi}{r^{3}} d \mu_{e}=-4 \pi \underset{r_{0}^{2}-|\vec{a}|^{2}}{|\vec{a}|}
$$

and

$$
\int_{S_{e}} \frac{1}{r^{3}} d \mu_{e}=4 \pi \underset{r_{0}^{2}-|\vec{a}|^{2}}{r_{0}} .
$$

Inserting then (5.18), (5.19) and (5.20) into (5.17) we arrive at the crucial inequality

$$
8 \pi m \frac{|\vec{a}|}{r_{0}} \leqq C H^{\varepsilon},
$$

where $C$ and $\varepsilon$ only depend on $q, m, C_{0}$. If we then fix $H_{1}$ so small that $|\vec{a}|<{ }_{2}^{1} r_{0}$ for all $H<H_{1}$, the minimum radius and the maximum radius on the surface $S$ are compatible. The case of surfaces which are far displaced, i.e. $|\vec{a}|>r_{0}$ can be treated similarly. The behaviour of such exterior surfaces under deformations has some interesting properties which will be treated elsewhere.

In particular we can now replace $\sigma_{0}^{-1}$ by $H$ in all our previous estimates. For instance we now get from Simons' identity the inequality

$$
\Delta|\AA| \geqq-\mathrm{CH}^{4},
$$

and an iteration as in the proof of Lemma 5.6 now yields the improved estimate

$$
|\AA| \leqq C H^{2},
$$

leading also to an improved estimate for the derivative of the curvature as in Lemma 5.7:

$$
|\nabla \AA| \leqq C H^{3} .
$$

It follows that the roundness estimates on the surface $S$ in (5.12) can be improved to

$$
\begin{aligned}
\left|\lambda_{i}{ }^{e}-r_{0}^{-1}\right| & \leqq C H^{2}, \quad i=1,2, \\
\left|(y-\vec{a})-r_{0} v_{e}\right| & \leqq C, \\
\left|v_{e}-r_{0}{ }^{-1}(y-\vec{a})\right| & \leqq C H .
\end{aligned}
$$

Carrying then out the computations for the displacement $|\vec{a}|$ above with these more precise estimates in mind we derive with $\varepsilon=1$ as in (5.21)

$$
8 \pi m \frac{|\vec{a}|}{r_{0}} \leqq C H
$$

which implies a uniform bound for $|\vec{a}|$ depending only on the given data $q, m, C_{0}$ provided $0<H<H_{1}$. Having thus obtained a uniform bound on the position of the surface keeping it close to a coordinate sphere it follows that the off diagonal parts of the Ricci curvature on $S$ satisfy the strong decay estimates stated in Lemma 3.8, Proposition 3.9 and Corollary 3.11. We can therefore 
proceed as in the parabolic case to finally get the estimates of Propositions 3.9 and 3.12:

$$
|\AA|^{2} \leqq C^{2} H^{6}, \quad|\nabla \AA|^{2} \leqq C^{2} H^{8} .
$$

Notice here that (5.22) and (5.23) guarantee the first two estimates in Lemma 3.8 .

The last two estimates above on the traceless part of the second fundamental form then show that for $\sigma_{0}$ sufficiently large, or equivalently, for $0<H<$ $H_{0}, H_{0}=H_{0}\left(q, m, C_{0}\right)$ sufficiently small, the surface $S$ is contained in some $B_{\sigma}\left(B_{1}, B_{2}, B_{3}\right)$, and Theorem 4.1 yields that $S$ has to coincide with the leaf of the foliation with mean curvature $H$. This completes the proof of Theorem 5.1.

Acknowledgement. This work was begun 1986 at the department of mathematics at UC San Diego, compare the annnouncement in [CY], and the authors would like to thank R.Schoen for very stimulating discussions during this time. The activities in San Diego triggered many further investigations into the subject, the authors particularly note an alternative proof for the existence part of our results by R.Ye in [Y].

\section{References}

[ADM] R. Arnowitt, S. Deser, C. Misner: Coordinate invariance and energy expressions in general relativity. Phys.Rev. 122 (1961) 997-1006.

[B1] R. Bartnik: The mass of an asymptotically flat manifold. Comm. Pure Appl. Math. 39 (1986) 661-693

[B2] R. Bartnik: Initial data for the Einstein equations in the quasi-spherical gauge, Gravitational Astronomy: Instrument Design and Astrophysical Prospects D.E. McLelland, H.A. Bachor, (ed.) World Scientific. (1990) 427-435

[CK] D. Christodoulou, S. Klainerman: The global nonlinear stability of the Minkowski space. Princeton Mathematical Series 41 (1993)

[CY] D. Christodoulou, S.T. Yau: Some remarks on the quasi-local mass, Contemporary Mathematics, Mathematics and General Relativity J. Isenberg (ed.) (1986) 9-14

[EH] K. Ecker, G. Huisken: Parabolic methods for the construction of spacelike slices of prescribed mean curvature in cosmological spacetimes. Comm. Math. Phys. 135 (1991) 595-613

[Hu1] G. Huisken: The volume preserving mean curvature flow. J.Reine Angew. Math. 382 (1987) 35-48

[Hu2] G. Huisken: Contracting convex hypersurfaces in Riemannian manifolds by their mean curvature, Invent. Math. 84 (1986) 463-480

[LY] P. Li, S.T. Yau: A new conformal invariant and its application to the Willmore conjecture and the first eigenvalue of compct surfaces. Invent. Math. 69 (1982). 269-291

[S] G. Stampacchia: Équations elliptiques du second ordre à coefficients discontinus, Montréal: Les Presses de l'Université, 1966.

[SSY] R. Schoen, L. Simon, S.T. Yau: Curvature estimates for minimal hypersurfaces. Acta Math. 134 (1975) 275-288

[SY1] R. Schoen, S.T. Yau: On the proof of the positive mass conjecture in General Relativity. Comm. Math. Phys. 65 (1979) 45-76

[SY2] R. Schoen, S.T. Yau: Proof of the positive mass theorem II. Comm. Math. Phys. 79 (1981) 231-260

[Y] R. Ye: Foliation by constant mean curvature spheres on asymptotically flat manifolds (Preprint) 